\title{
Synthesis of some new pyrazolo[1,5-a] pyrimidine, pyrazolo[5,1-c]triazine, 1,3,4-thiadiazole and pyridine derivatives containing 1,2,3-triazole moiety
}

\author{
Nadia A. Abdelriheem ${ }^{1}$, Yasser H. Zaki ${ }^{2,3^{*}}$ and Abdou O. Abdelhamid
}

\begin{abstract}
Background: Pyrazolo[1,5-a]pyrimidines are purine analogues. They have beneficial properties as antimetabolites in purine biochemical reactions. This division compounds have attracted wide pharmaceutical interest because of their antitrypanosomal activity.

Results: The present work depicts an effective synthesis convention of pyrazolo[1,5-a]pyrimidines, pyrazolo[5,1-c] triazines, thieno[2,3-b]pyridines and polysubstituted pyridines containing 1,2,3,-triazole moiety from the reaction of sodium 3-(5-methyl-1-(p-toly)-1 H-1,2,3-triazol-4-yl)-3-oxoprop-1-en-1-olate with the fitting heterocyclic amines and its diazonium salt, and active methylene compounds, individually. Likewise, thiazoles and, 1,3,4-thiadiazoles were obtained from 2-bromo-1-(5-methyl-1-( -tolyl)-1H-1,2,3-triazol-4-yl)ethanone and some reagent such as hydrazonoyl chlorides and halo ketones. The newly synthesized compounds were established by elemental analysis, spectral data, and alternative synthetic route whenever possible.

Conclusions: New series of pyrazolo[1,5-a]pyrimidines, pyrazolo[5,1-c]triazines, thieno[2,3-b]pyridines and polysubstituted pyridines containing the 1,2,3,-triazole moiety were synthesized via reactions of sodium 3-(5-methyl-1-( $p$ toly)-1H-1,2,3-triazol-4-yl)-3-oxoprop-1-en-1-olate with the appropriate heterocyclic amines and its diazonium salt. In addition, 1,3,4-thiadiazoles and, 1,3-thiazoles were acquired in a decent yield via the reaction of substituted thiourea with the appropriate hydrazonoyl chlorides and halogenated ketenes.
\end{abstract}

Keywords: 1,2,3-Triazole, Pyrazolo[1,5-a]pyrimidines, Pyrazolo[5,1-c]triazines, Thieno[2,3-b]pyridines, 1,3,4-Thiadiazoles, Hyrazonoyl chlorides, Thiazoles, Pyridines

\section{Background}

Pyrazolo[1,5-a]pyrimidines are purine analogs and therefore have valuable properties as antimetabolites in purine biochemical activity. This class of compounds has attracted wide pharmaceutical interest because of their antitrypanosomal activity [1], antischistosomal activity [2], and other activities such as HMG-CoA reductase inhibitors [3], COX-2 selective inhibitors [4], AMP phosphodiesterase inhibitors [5], KDR kinase inhibitors [6],

\footnotetext{
*Correspondence: yzaki2002@yahoo.com

${ }^{2}$ Department of Chemistry, Faculty of Science, Beni-Suef University, Beni-Suef 62514, Egypt

Full list of author information is available at the end of the article
}

selective peripheral benzodiazepine receptor ligaments [7], antimicrobial agents [8], and as antianxiety agents [9]. Recently other pharmaceutical activities have been reported, for example, as an agent for the treatment of sleep disorders [10] and as an oncological agent [6]. Also, pyrazolo[5,1-c][1,2,4]triazines are known to exhibit a broad range of biological activities [11-15]. Due to their structural similarities to nucleic bases, pyrazolo[5,1-c] $[1,2,4]$ triazines may act as metabolites and therefore they can be useful as antiviral and antitumor agents [11]. Pyrazolotriazines have indicated a remarkable cytotoxic activity against colon, breast, and lung carcinoma cells [16]. Some derivatives showed selective cytotoxicity in 
hypoxic and normoxic conditions [17]. The 1,3,4-thiadiazole derivatives have attracted considerable interest due to their wide spectra of biological activities such as antibacterial, antifungal, antituberculosis, anti-hepatitis B viral, antileishmanial, anti-inflammatory, analgesic, CNS depressant, anticancer, antioxidant, antidiabetic, molluscicidal, antihypertensive, diuretic, analgesic, antimicrobial, antitubercular, and anticonvulsant activities [18-27].

\section{Results and discussion \\ Chemistry}

The reaction of 1-(5-methyl-1-( $p$-tolyl)-1H-1,2,3-triazol-4-yl)ethan-1-one (1) with ethyl formate in diethyl ether in the presence of sodium methoxide has afforded sodium 3-(5-methyl-1-( $p$-tolyl)-1H-1,2,3-triazol-4-yl)3-oxoprop-1-en-1-olate (2). Likewise, compound (1) reacted with $\mathrm{N}, \mathrm{N}$-dimethylformamide-dimethylacetal in boiling xylene to afford 3-(dimethylamino)-1-(5methyl-1-( $p$-tolyl)-1H-1,2,3-triazol-4-yl)prop-2-en-1one (6). The reactivity of compound (2) and compound (6) towards heterocyclic amines was inspected. In this manner, reaction of compound (2) or compound (6) with each of 3-amino-5-phenylpyrazole (3a), 3-amino4-phenylpyrazole (3b), 3-amino-4-cyanopyrazole (3c), 3-amino-1,2,4-triazole (3d), 2-aminobenzimidazole (3e) and 4,6-dimethyl-2H-pyrazolo[3,4-b]pyridin-3-amine (3f) in refluxing piperidinium acetate, in each case, only one isolable product as evidenced by TLC. The isolated products $(\mathbf{5 a}-\mathbf{f})$ (Scheme 1) were identified, on the base of their elemental analysis, spectral data and according to similar data obtained before [28-30].

The reaction of compound (2) or compound (6) with each of diazotized 3-amino-5-phenylpyrazole $(\mathbf{8 a})$ and diazotized 3-amino-4-phenylpyrazole $(\mathbf{8 b})$ in ethanol containing sodium acetate at $0-5{ }^{\circ} \mathrm{C}$ yielded products that were distinguished as (5-methyl-1-( $p$-tolyl)- $1 \mathrm{H}$ 1,2,3-triazol-4-yl) (7-phenylpyrazolo [5,1-c] $[1,2,4]$ triazin-3-yl)-methanone (10a) and (5-methy-1-( $p$-tolyl)$1 H$-1,2,3-triazol-4-yl)(8-phenylpyrazolo[5,1-c] [1,2,4] triazin-3-yl)-methanone (10b), respectively (Scheme 2 ). The structures of the products $(\mathbf{1 0 a})$ and $(\mathbf{1 0 b})$ were consistent with their elemental and spectral (Ms, IR, ${ }^{1} \mathrm{H}$ NMR, and the ${ }^{13} \mathrm{C}$ NMR) analysis (see "Experimental section"). To account for the formation of the products 10a and $\mathbf{1 0 b}$, it is suggested as depicted in (Scheme 2) that the reaction start with electrophilic substitution to yield the corresponding azo derivative, which undergoes in situ dehydrative cyclization, gave the corresponding $\mathbf{1 0}$ as a final product.

Treatment of compound (2) with each of benzenediazonium chloride (11a) or $p$-toluidine diazonium chloride $(\mathbf{1 1 b})$ in ethanol containing sodium acetate as a buffer solution yielded 3-(5-methyl-1-
(p-tolyl)-1H-1,2,3-triazol-4-yl)-3-oxo-2-(2-phenylhydrazono)propanal (12a), 3-(5-methyl-1-(p-tolyl)-1H-1,2,3triazol-4-yl)-3-oxo-2-(2-( $p$-tolyl)hydrazono)propanal (12b), respectively (Scheme 3). The structures of compound (12a) and compound (12b) were affirmed by elemental analysis, spectral data, and alternative synthetic route. In this way, 3-(dimethylamino)-1-(5-methyl-1(p-tolyl)-1H-1,2,3-triazol-4-yl)prop-2-en-1-one (6) was coupled with benzenediazonium chloride or $p$-toluidinediazonium chloride to give a product indistinguishable in all aspects (m.p., mixed m.p. and spectra) with compound (12a) and compound (12b), respectively. The ${ }^{1} \mathrm{H}$ NMR spectrum of compound (12a) showed signals at $\delta=2.06$ (s, 3H, $\left.\mathrm{CH}_{3}\right), 2.34\left(\mathrm{~s}, 3 \mathrm{H}, 4-\mathrm{CH}_{3} \mathrm{C}_{6} \mathrm{H}_{4}\right), 7.26-8.20(\mathrm{~m}, 9 \mathrm{H}$, ArH's), 9.75 (s, 1H, CHO) and 14.39 (s, br., $\mathrm{NH}$ ).

Reaction of compound (2) with cyanothioacetamide (13) in piperdinium acetate gave 2-mercapto-6-(5methyl-1-( $p$-tolyl)-1H-1,2,3-triazol-4-yl)nicotinonitrile (14). The Structure of compound (14) was elucidated by elemental analysis, spectral data, and alternative synthetic route or chemical transformation. Thus, treatment of compound (6) with cyanothioacetamide in ethanol containing a catalytic amount of piperidine under reflux gave a product identical in all aspects (m.p., mixed m.p. and spectra) with compound (14). The product formulated from treatment of compound (14) with ethyl chloroacetate, in $\mathrm{N}, \mathrm{N}$-dimethylformamide containing potassium hydroxide was ethyl 3-amino-6-(5methyl-1-( $p$-tolyl)-1H-1,2,3-triazol-4-yl)thieno[2,3- $b$ ] pyridine-2-carboxylate (15a) corresponding to the addition, dehydrochlorination, and cyclization reactions (Scheme 4). IR spectrum of compound (15a) showed a band at 3460, $3355\left(\mathrm{NH}_{2}\right.$ group) and no band of the $\mathrm{CN}$ function between 2100 and $2300 \mathrm{~cm}^{-1}$. The ${ }^{1} \mathrm{H}$ NMR spectrum of compound (15a) revealed signals at $1.26(\mathrm{t}$, $\left.3 \mathrm{H}, J=7 \mathrm{~Hz}, \mathrm{CH}_{2} \mathrm{CH}_{3}\right), 2.34\left(\mathrm{~s}, 3 \mathrm{H}, 4-\mathrm{CH}_{3} \mathrm{C}_{6} \mathrm{H}_{4}\right), 2.64$ (s, $\left.3 \mathrm{H}, \mathrm{CH}_{3}\right), 4.23\left(\mathrm{q}, 2 \mathrm{H}, J=7 \mathrm{~Hz}, \mathrm{CH}_{2} \mathrm{CH}_{3}\right), 6.8$ (s, br., $\left.2 \mathrm{H}, \mathrm{NH}_{2}\right), 7.32-7.63(\mathrm{~m}, 5 \mathrm{H}, \mathrm{ArH}$ 's) and $8.81-8.83(\mathrm{~d}, 1 \mathrm{H}$, $\mathrm{ArH}$ ) and absence of signals of the $-\mathrm{SCH}_{2}-$ group. These results proved that the $\mathrm{CN}$ and the $-\mathrm{SCH}_{2}-$ groups were both involved in the cyclization step leading to compound (15a).

Also, compound (14) was reacted with each of chloroacetone and $\omega$-bromoacetophenone in $N, N$-dimethylformamide containing potassium hydroxide to afford 1-(3-amino-6-(5-methyl-1-( $p$-tolyl)-1H-1,2,3-triazol4-yl)thieno[2,3-b]pyridin-2-yl)ethan-1-one $\quad(\mathbf{1 5 b})$ and 6-(3-amino-6-(5-methyl-1-( $p$-tolyl) - $1 H-1,2,3$-triazol-4-yl)thieno[2,3-b]pyridin-2-yl)(phenyl)methanone $(\mathbf{1 5 c})$ respectively. Similarly, compound (14) was reacted with chloroacetonitrile afforded 3-amino-6-(5methyl-1-( $p$-tolyl)-1H-1,2,3-triazol-4-yl)thieno[2,3-b] pyridine-2-carbonitrile (16), in a good yield (Scheme 4). 


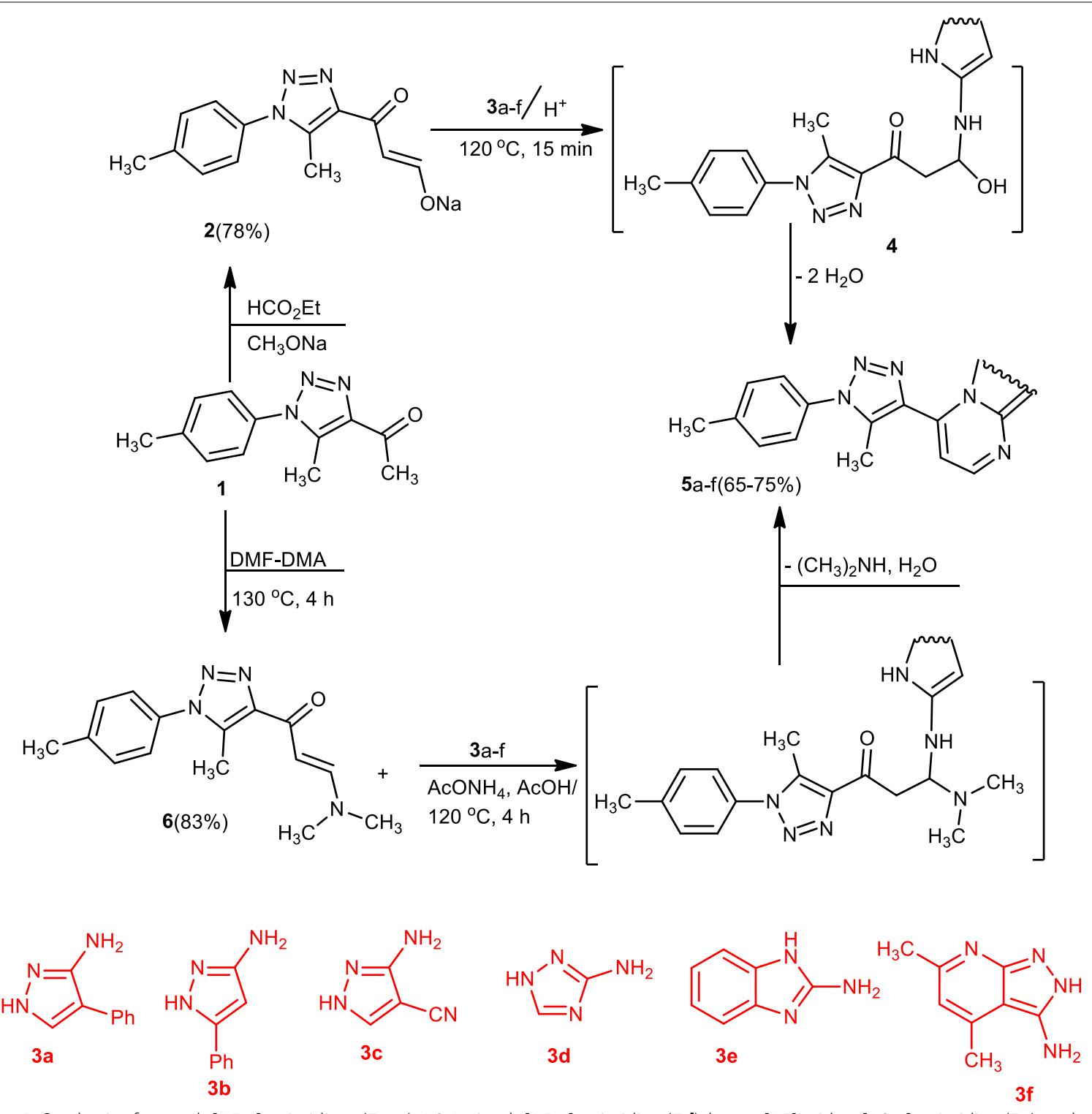

Scheme 1 Synthesis of pyrazolo[1,5-a]pyrimidines (5a-c), 1,2,4-triazolo[1,5-a]pyrimidine (5d), benzo [4,5]imidazo[1,2-a]pyrimidine (5e), and pyrido $\left[2^{\prime}, 3^{\prime}: 3,4\right]$ pyrazolo[1,5-a]pyrimidine $(\mathbf{5 f})$

The structures of compounds $(\mathbf{1 5 a}-\mathbf{c})$ and (16) were confirmed by elemental analysis and spectral data. Treatment of compound (6) with each of ethyl acetoacetate, acetylacetone, ethyl cyanoacetate, malononitrile or benzoylacetonitrile in boiling acetic acid containing ammonium acetate under reflux gave ethyl 2-methyl-6-(5-methyl-1- $p$-tolyl-1H-1,2,3-triazol4-yl)pyridine-3-carboxylate (17), 1-(2-methyl-6-(5methyl-1- $p$-tolyl-1H-1,2,3-triazol-4-yl)pyridin-3-yl) ethanone (18), 1,2-dihydro-6-(5-methyl-1-p-tolyl-1 $H$ 1,2,3-triazol-4-yl)-2-oxopyridine-3-carbonitrile 2-amino-6-(5-methyl-1-p-tolyl-1H-1,2,3-triazol-4-yl) pyridine-3-carbonitrile (21), 6-(5-methyl-1-( $p$-tolyl)- $1 H$ 1,2,3-triazol-4-yl)pyridin-3-phenyl-2-carbonitrile (22), respectively (Scheme 5). Structures (17), (18), and (2022) were confirmed based on elemental analysis and spectral data (cf. "Experimental section").

Next, 4-(5-methyl-1-( $p$-tolyl)-1H-1,2,3-triazol-4-yl) thiazol-2-amine (25) was prepared from the reaction of 2-bromo-1-(5-methyl-1-( $p$-tolyl)-1H-1,2,3-triazol-4-yl) ethanone (23) [31] with thiourea. The structure of compound (25) was established based on elemental analysis, spectral data, and chemical transformation. Thus, compound (25) was coupled with 


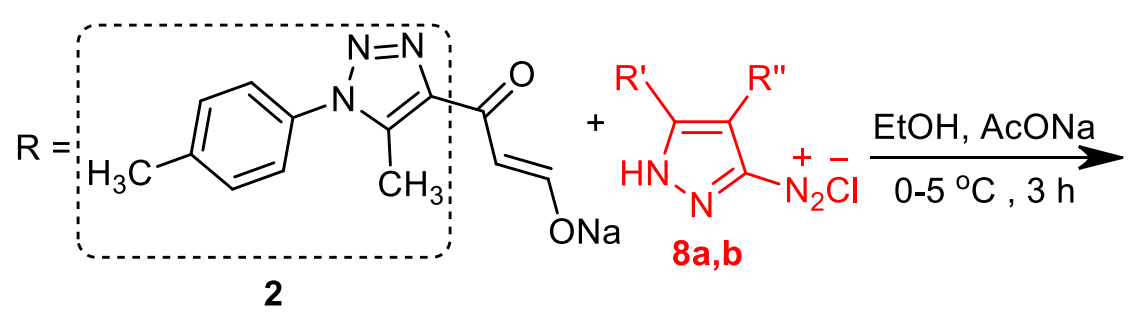<smiles>[R]C(=O)C(C)N=Nc1nn2c([R])c1CN([R]([H])=C(C)CO)N2</smiles>

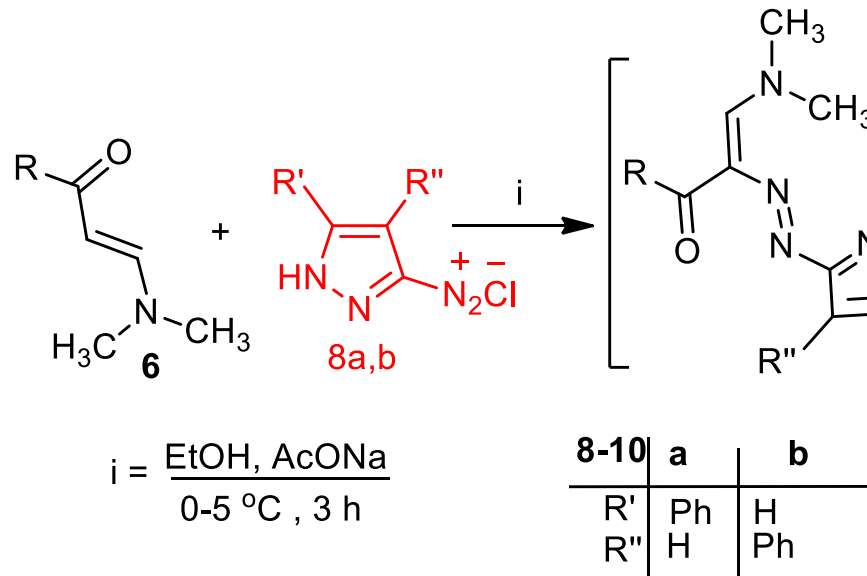<smiles>[R]C(=O)c1cn2nc([R])c([R7])c2nn1</smiles>

$10 \mathrm{a}, \mathrm{b}(70 \%, 75 \%)$

Scheme 2 Synthesis of pyrazolo[5,1-c]triazines (10)

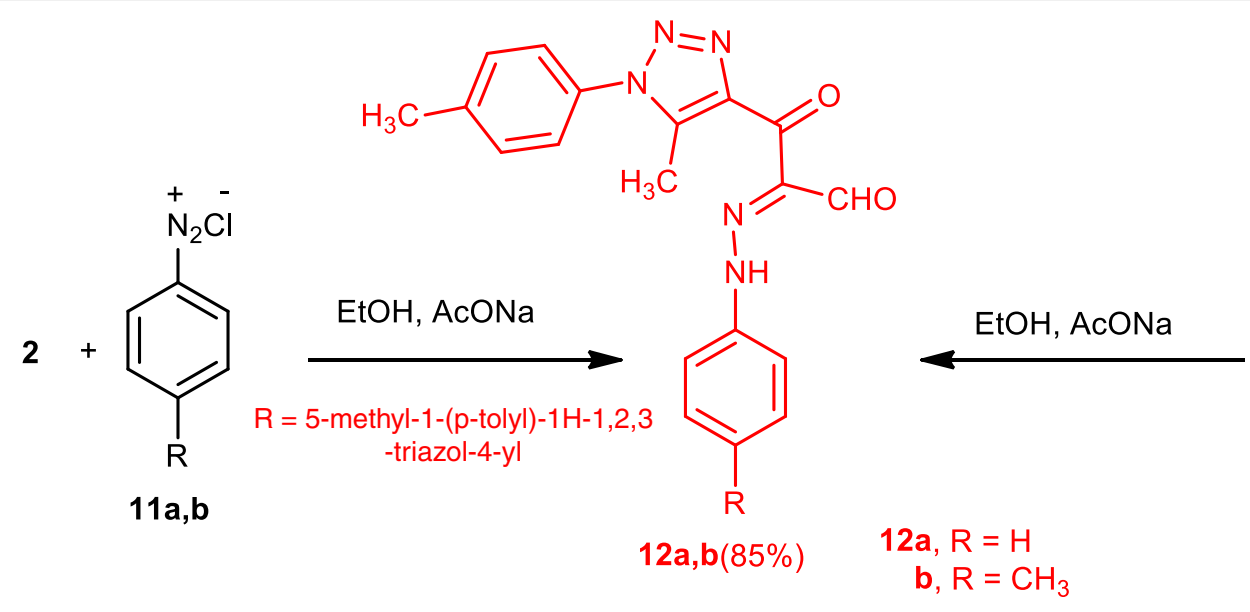

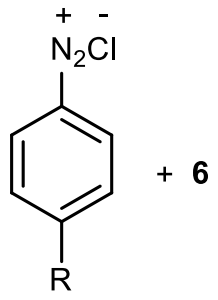

$11 a, b$

Scheme 3 Synthesis of 3-(5-methyl-1-(p-tolyl)-1H-1,2,3-triazol-4-yl)-3-oxo-2-(2-(aryl)hydrazono)propanal (12a) and (12b)

arenediazonium chlorides in ethanol contained sodium acetate to afford 4-(5-methyl-1-( $p$-tolyl)-1H-1,2,3-triazol-4-yl)-5-(phenyldiazenyl)thiazol-2-amine (26a) and 5-((4-chlorophenyl)diazenyl)-4-(5-methyl-1-( $p$-tolyl)$1 H$-1,2,3-triazol-4-yl)thiazol-2-amine (26b), respectively (Scheme 6). More evidence on the correct structure of compound (26a) was obtained via reaction of thiourea with 2-(5-methyl-1-( $p$-tolyl)-1H-1,2,3-triazol-4-yl)- 2-oxo- $N$-phenylacetohydrazonoyl bromide (28) in boiling ethanol (cf. "Experimental section").

1-(4-(5-Methyl-1-( $p$-tolyl)-1H-1,2,3-triazol-4-yl)thiazol-2-yl)-3-phenylthiourea (27) was prepared via reaction of compound (25) with phenyl isothiocyanate in $\mathrm{N}, \mathrm{N}$-dimethylformamide containing potassium hydroxide, followed by acidification with hydrochloric acid. The structure of compound (27) was confirmed by elemental 

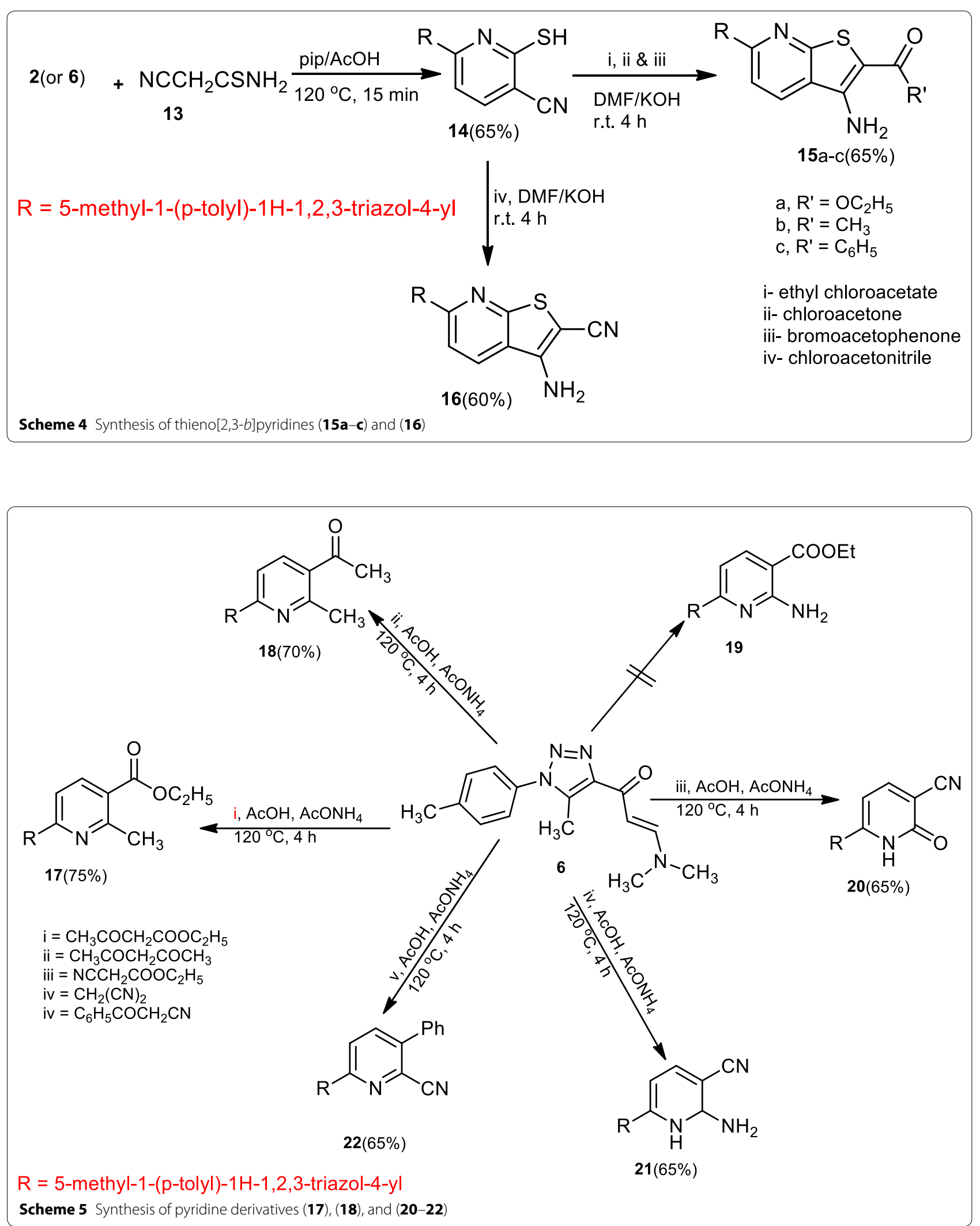


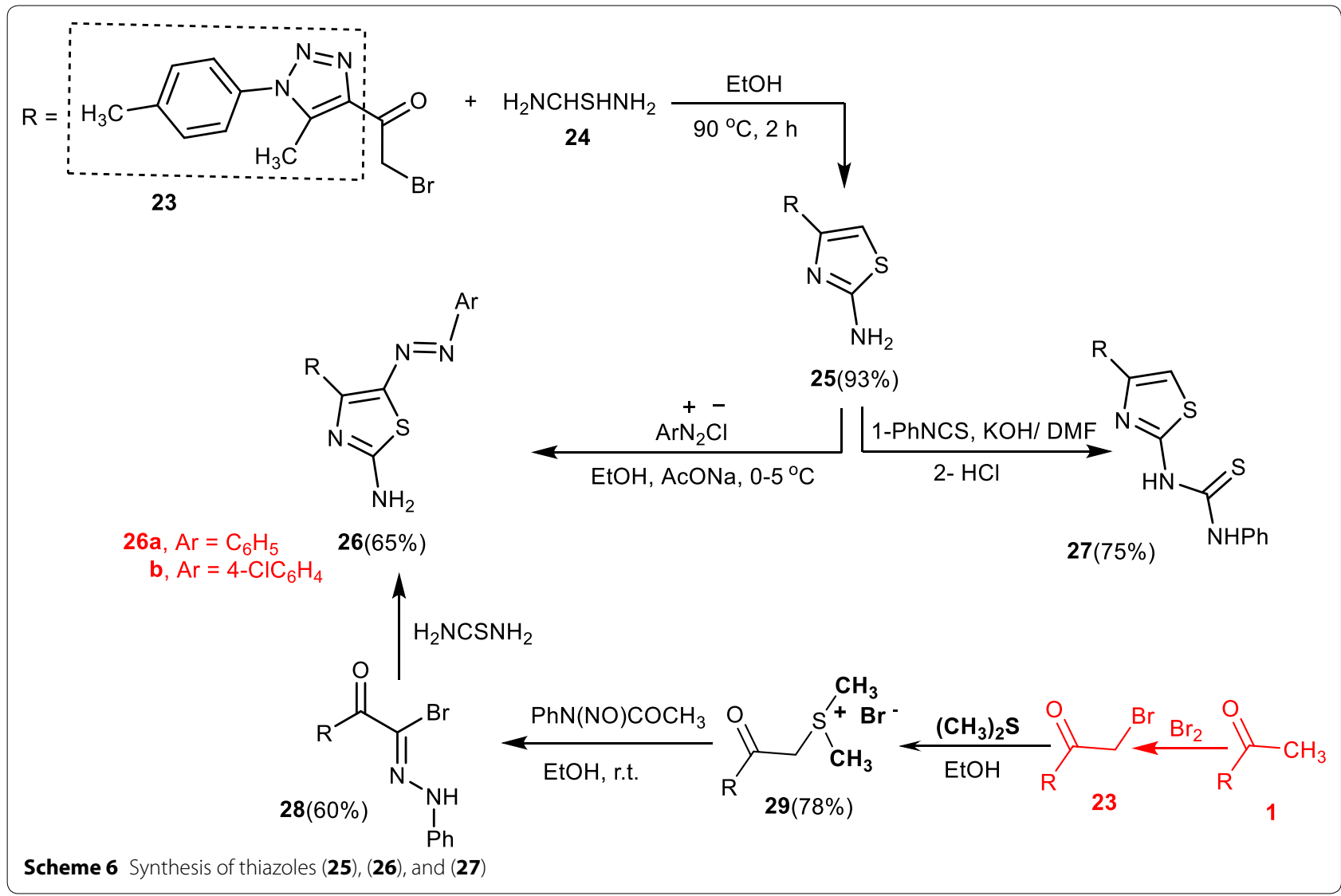

analysis, spectral data, and chemical transformation. Thus, the appropriate hydrazonoyl chloride (30a-d) were reacted with thioanilide (27) in $N, N$-dimethylformamide in presence of triethylamine or potassium hydroxide to give one isolable product according to TLC. The structure of the product may be one from the structure of compound (31), (31A) or (31B). The obtained spectral data, however, compatible only with the structures of $(\mathbf{3 1 a}-\mathbf{d})$ and formulated as: $N$-(3-aryl-5-substituted-1,3,4-thiadiazol-2(3H)-ylidene)-4-(5-methyl-1-( $p$-tolyl)- $1 H$-1,2,3-triazol-4-yl)thiazol-2-amine (31a-d) (Scheme 7).

Treatment of thiourea derivative (27) with $\omega$-bromoacetophenone or ethyl chloroacetate in refluxing ethanol in the presence of triethylamine gave $N$-(3,4-diphenylthiazol-2(3H)-ylidene)-4-(5-methyl1 -(p-tolyl)-1H-1,2,3-triazol-4-yl)thiazol-2-amine (32) and 2-((4-(5-methyl-1-(p-tolyl)-1H-1,2,3-triazol-4-yl)-thiazol2-yl)imino)-3-phenylthiazolidin-4-one (33), respectively (Scheme 8).

\section{Experimental section} General methods

All melting points were determined on an electro thermal Gallen Kamp melting point apparatus (lain George,
Calgary, Canda) and are uncorrected. IR $\left(\mathrm{cm}^{-1}\right)$ spectra were recorded on $\mathrm{KBr}$ disk on a FTIR-8201 spectrophotometer (Shimadzu, Tokyo, Japan). ${ }^{1} \mathrm{H}$ NMR and ${ }^{13} \mathrm{C}$ NMR spectra were measured in deuterated dimethyl sulfoxide (DMSO-d6) using a Mercury VX-300 NMR spectrometer (Varian, Inc., Palo Alto, California 94304 USA). Mass spectra were recorded on a Shimadzu GCMS-QP1000 EX mass spectrometer (Tokyo, Japan) at $70 \mathrm{eV}$. Measurements of the elemental analysis were carried out at the Microanalytical Centre of Cairo University, Giza, Egypt. All reactions were followed by TLC (Silica gel, Merck, Kenilworth, NJ, USA). Hydrazonoyl halides were prepared as previously reported [32, 33].

\section{Synthesis of sodium salt of 3-hydroxy-1-(5-methyl-1-(p-tolyl)-} $1 \mathrm{H}$-1,2,3-triazol-yl)prop-2-en-1-one (2)

A solution of 1-(5-methyl-1-(p-tolyl)- $1 H$-1,2,3-triazol4-yl)ethan-1-one (1) [34], (5.4 g, $25 \mathrm{mmol})$ in ether $(25 \mathrm{ml})$ was added to a mixture of sodium methoxide (1.4 g, $25 \mathrm{mmol})$ and ethyl formate $(1.9 \mathrm{ml}, 25 \mathrm{mmol})$ in dry ether $(25 \mathrm{ml})$ while stirring in ice-bath at $0-5{ }^{\circ} \mathrm{C}$ for $2 \mathrm{~h}$. The resulting solid was collected and washed with diethyl ether which afforded compound (2) that was used without crystallization, yield (76\%). 


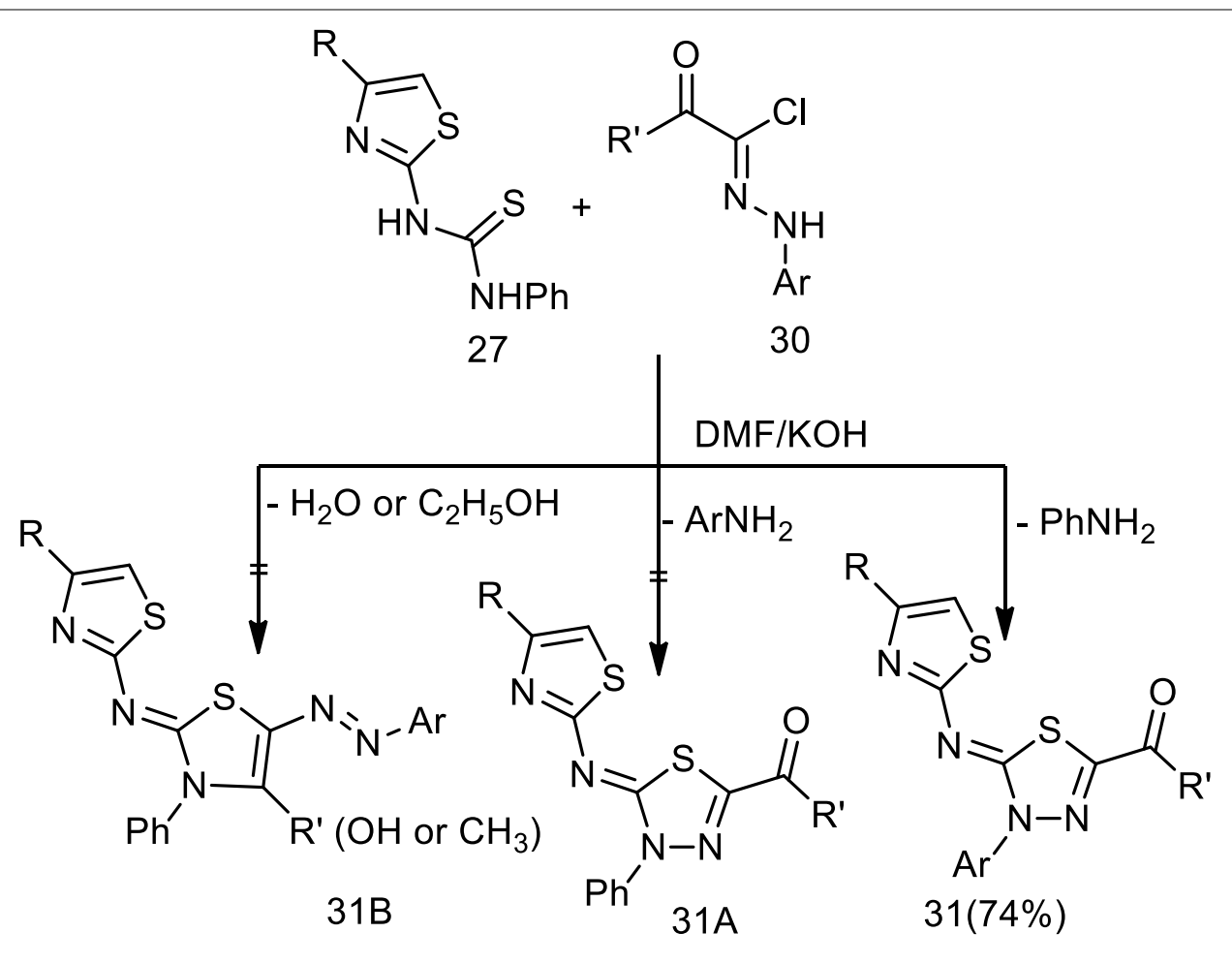

$\mathrm{R}=$ 5-methyl-1-(p-tolyl)-1H-1,2,3-triazol-4-yl

$$
\begin{array}{rlrl}
30,31 \mathrm{a}, \mathrm{R}^{\prime} & =\mathrm{C}_{2} \mathrm{H}_{5} \mathrm{O}, \mathrm{Ar} & =\mathrm{C}_{6} \mathrm{H}_{5} \\
\text { b, } \mathrm{R}^{\prime} & =\mathrm{C}_{2} \mathrm{H}_{5} \mathrm{O}, \mathrm{Ar}=4-\mathrm{CH}_{3} \mathrm{C}_{6} \mathrm{H}_{4} \\
\text { c, } \mathrm{R}^{\prime} & =\mathrm{CH}_{3}, & \mathrm{Ar} & =\mathrm{C}_{6} \mathrm{H}_{5} \\
\text { d, } \mathrm{R}^{\prime} & =\mathrm{CH}_{3}, & \mathrm{Ar} & =4-\mathrm{CH}_{3} \mathrm{C}_{6} \mathrm{H}_{4}
\end{array}
$$

Scheme 7 Synthesis of 1,3,4-thiadiazoles (31a-d)

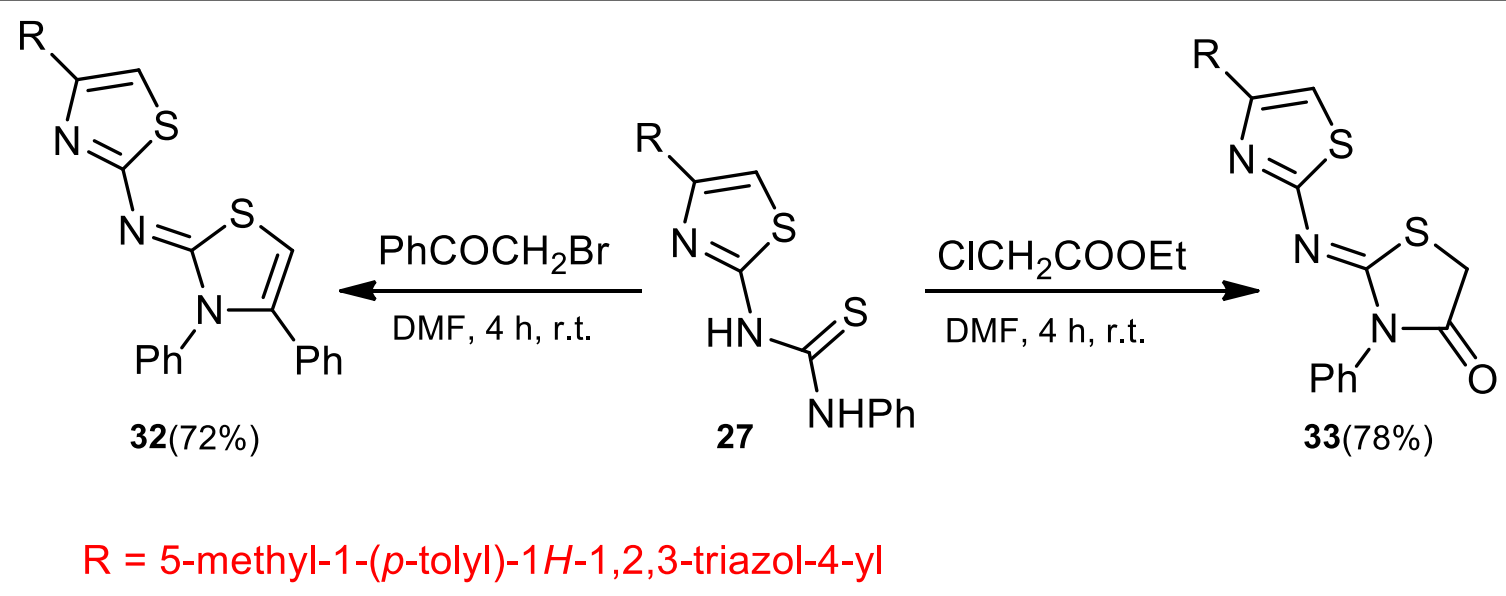

Scheme 8 Synthesis of thiazole (32) and thiazolone (33) 
Synthesis of 3-(dimethylamino)-1-(5-methyl-1-(p-tolyl)-1H-1, 2,3-triazol-4-yl)prop-2-en-1-one (6)

A mixture of 1-(5-methyl-1-( $p$-tolyl)- $1 \mathrm{H}$-1,2,3-triazol4-yl)ethane-1-one (1) (2.3 g, $0.1 \mathrm{~mol})$ and N,N-dimethylformamide-dimethylacetal $(11.9 \mathrm{~g}, 14 \mathrm{ml}, 0.1 \mathrm{~mol})$ in dry xylene $(30 \mathrm{ml})$ was heated under reflux for $4 \mathrm{~h}$. The hot solution evaporated to its half volume and then cooled. The resulting solid was collected and recrystallized from benzene to give the compound (6) as orange crystals. Yield: (83\%); m.p. b135 ${ }^{\circ} \mathrm{C}$. FT-IR $\left(\mathrm{KBr}, \mathrm{cm}^{-1}\right)$ : 3041, 2965 (CH), 1688 (CO), $1645(\mathrm{C}=\mathrm{N}), 1589(\mathrm{C}=\mathrm{C}) ;{ }^{1} \mathrm{H}$ NMR (300 MHz, DMSO-d6): $\delta=2.31\left(\mathrm{~s}, 3 \mathrm{H}, \mathrm{CH}_{3}\right), 2.42(\mathrm{~s}, 3 \mathrm{H}$, $\left.\mathrm{CH}_{3}\right), 2.48\left(\mathrm{~s}, 3 \mathrm{H}, \mathrm{CH}_{3}\right), 3.15\left(\mathrm{~s}, 3 \mathrm{H}, \mathrm{CH}_{3}\right), 6.15(\mathrm{~d}, 1 \mathrm{H}$, $J=12 \mathrm{~Hz}, \mathrm{CH}=), 7.76$ (d, $1 \mathrm{H}, J=12 \mathrm{~Hz}, \mathrm{CH}=)$; 7.407.50 (m, 4H, ArH's). Anal. Calcd. for $\mathrm{C}_{15} \mathrm{H}_{18} \mathrm{~N}_{4} \mathrm{O}(270.34)$, C, 66.64; H, 6.71; N, 20.73. Found: C, 66.67; H, 6.69; N, 20.80 .

\section{Synthesis of pyrazolo[1,5-a]pyrimidines (5a-c), [1,2,4] triazolo[1,5-a]pyrimidine (5d), benzo [4,5]imidazo[1,2-a] pyrimidine (5e) and pyrido[2',3':3,4]pyrazolo[1,5-a]pyrimi- dine (5f)}

Method A A mixture of sodium salt (2) (1.32 g, $10 \mathrm{mmol})$ and the appropriate heterocyclic amines $(3 \mathbf{a}-\mathbf{f})(10 \mathrm{mmol})$ in a solution of piperidinium acetate [piperidine $(2.5 \mathrm{ml})$, water $(5 \mathrm{ml})$ and acetic acid $(2 \mathrm{ml})]$ was heated under reflux for $15 \mathrm{~min}$, acetic acid $(1.5 \mathrm{ml})$ was added to the reaction mixture while boiling, then the mixture was cooled and the resulting solid was collected and crystallized from the proper solvent gave $(\mathbf{5 a}-\mathbf{f})$.

Method B A mixture of compound (6) (1.35 g, $10 \mathrm{mmol})$, the appropriate heterocyclic amines $(\mathbf{3 a}-\mathbf{f})(10 \mathrm{mmol})$ and ammonium acetate $(0.77 \mathrm{~g}, 10 \mathrm{mmol})$ in acetic acid $(20 \mathrm{ml})$ was heated under reflux for $4 \mathrm{~h}$. The reaction mixture was cooled, after that, the resulting solid was collected and crystallized from the proper solvent and gave product identical in all aspects (m.p., mixed m.p., spectra) with the corresponding $(\mathbf{5 a}-\mathbf{f})$, which was obtained in method A.

\section{7-(5-Methyl-1-(p-tolyl)-1H-1,2,3-triazol-4-yl)-2-phenylpyrazol o[1,5-a]pyrimidine (5a)}

Yellow crystals from ethanol, yield (75\%); m.p. 195$197^{\circ} \mathrm{C}$. FT-IR (KBr, cm $\left.{ }^{-1}\right): 2981(\mathrm{CH}) ; 1635(\mathrm{C}=\mathrm{N}) ; 1566$ $(\mathrm{C}=\mathrm{C}) .{ }^{1} \mathrm{H} \mathrm{NMR}\left(300 \mathrm{MHz}, \mathrm{CDCl}_{3}\right): \delta=2.50(\mathrm{~s}, 3 \mathrm{H}$, $\left.\mathrm{CH}_{3}\right) 2.65\left(\mathrm{~s}, 3 \mathrm{H}, \mathrm{CH}_{3}\right), 6.82(\mathrm{~s}, 1 \mathrm{H}$, pyrazol H-4), $7.13(\mathrm{~d}$, $1 \mathrm{H}, J=4 \mathrm{~Hz}$, pyrimide H-5), 7.32-7.35 (m, 2H, ArH's), 7.45-7.62 (m, 5H, ArH's), 7.77-7.82 (m, 2H, ArH's), $8.57(\mathrm{~d}, 1 \mathrm{H}, J=4 \mathrm{~Hz}$, pyrimide $\mathrm{H}-6) .{ }^{13} \mathrm{C} \mathrm{NMR}\left(\mathrm{CHCl}_{3}\right)$ $\delta=10.4,20.6$, 98.8, 111.2, 122.5, 127.4, 128.4, 128.8, $130.1,131.8,132.2,133.4,139.7,141.2,144.5,146.4$, 148.2, 152.3. Anal. Calcd. for $\mathrm{C}_{22} \mathrm{H}_{18} \mathrm{~N}_{6}$ (366.43): C, 72.11; H, 4.95; N, 22.94. Found: C, 72.20; H, 4.80; N, 22.89.
7-(5-Methyl-1-(p-tolyl)-1H-1,2,3-triazol-4-yl)-3-phenylpyrazol $o[1,5$-a]pyrimidine (5b)

Yellow crystals from ethanol, yield (75\%); m.p. $230{ }^{\circ} \mathrm{C}$. FT-IR (KBr, cm $\left.{ }^{-1}\right)$ : $3028(\mathrm{CH}) ; 1635(\mathrm{C}=\mathrm{N}) ; 1573(\mathrm{C}=\mathrm{C})$. ${ }^{1} \mathrm{H}$ NMR (300 MHz, $\left.\mathrm{CDCl}_{3}\right): 2.49\left(\mathrm{~s}, 3 \mathrm{H}, \mathrm{CH}_{3}\right) 2.59$ (s, $3 \mathrm{H}, \mathrm{CH}_{3}$ ), 6.90-6.92 (d, 2H, $J=8 \mathrm{~Hz}$, ArH's), 7.10 (d, $1 \mathrm{H}, J=8 \mathrm{~Hz}$, pyrimidine $\mathrm{H}-5$ ), 7.32-7.35 (m, 2H, ArH's), 7.45-762 (m, 5H, ArH's), 8.32 (s, 1H, pyrazole $\mathrm{H}-3$ ), and $8.68(\mathrm{~d}, 1 \mathrm{H}, J=4 \mathrm{~Hz}$, pyrimidine). Anal. Calcd. for $\mathrm{C}_{22} \mathrm{H}_{18} \mathrm{~N}_{6}$ (366.43): C, 72.11; H, 4.95; N, 22.94. Found: C, $72.20 ; \mathrm{H}, 4.80 ; \mathrm{N}, 22.89$.

\section{7-(5-Methyl-1-(p-tolyl)-1H-1,2,3-tria-}

zol-4-yl)-pyrazolo[1,5-a]pyrimidin-3-carbonitrile (5c)

Orange crystals from ethanol, yield (70\%); m.p. 235$237^{\circ} \mathrm{C}$. FT-IR (KBr, cm $\left.{ }^{-1}\right)$ : 3039, $2970(\mathrm{CH}) ; 2225(\mathrm{CN})$; $1635(\mathrm{C}=\mathrm{N}) ; 1573(\mathrm{C}=\mathrm{C}) .{ }^{1} \mathrm{H}$ NMR $\left(300 \mathrm{MHz}, \mathrm{CDCl}_{3}\right)$ : $\delta=2.49\left(\mathrm{~s}, 3 \mathrm{H}, \mathrm{CH}_{3}\right) 2.54\left(\mathrm{~s}, 3 \mathrm{H}, \mathrm{CH}_{3}\right), 7.26-7.59(\mathrm{~m}$, $5 \mathrm{H}, \mathrm{ArH}$ ), 8.95 (s, $1 \mathrm{H}$, pyrazol $\mathrm{H}-3)$, and $8.84(\mathrm{~d}, 1 \mathrm{H}$, $J=4 \mathrm{~Hz}$, pyrimidine $\mathrm{H}-6) .{ }^{13} \mathrm{C}$ NMR in $\mathrm{CHCl}_{3} \delta=10.4$, 20.6, 98.8, 52.4 (CN), 111.2, 11.3.1, 122.4, 128.4, 133.4, 135.1, 139.7, 141.2, 144.5, 146.4, 148.2, 155.3. Anal. Calcd. for $\mathrm{C}_{17} \mathrm{H}_{13} \mathrm{~N}_{7}$ (315.39): C, 64.75; H, 4.16; N, 31.09. Found: C, 64.65; H, 4.26; N, 31.12.

\section{5-(5-Methyl-1-(p-tolyl)-1H-1,2,3-triazol-4-yl) [1,2,4] triazolo[1,5-a]pyrimidine (5d)}

White crystals from acetic acid, yield (65\%); m.p. $302{ }^{\circ} \mathrm{C}$. FT-IR (KBr, cm $\left.{ }^{-1}\right)$ : 3047, $2993(\mathrm{CH}) ; 1620(\mathrm{C}=\mathrm{N}), 1577$ $(\mathrm{C}=\mathrm{C}) .{ }^{1} \mathrm{H}$ NMR $(300 \mathrm{MHz}, \mathrm{DMSO}-\mathrm{d} 6): \delta=2.07(\mathrm{~s}$, $\left.3 \mathrm{H}, \mathrm{CH}_{3}\right) 2.49\left(\mathrm{~s}, 3 \mathrm{H}, \mathrm{CH}_{3}\right), 6.62-6.63(\mathrm{~d}, J=4 \mathrm{~Hz}, 1 \mathrm{H}$, pyrimidine $\mathrm{H}-5), 7.14-7.67(\mathrm{~m}, 4 \mathrm{H}, \mathrm{ArH}, \mathrm{s}), 8.27$ (s, 1H, triazole), 9.27-9.28 (d, $1 \mathrm{H}, J=4 \mathrm{~Hz}$, pyrimidine $\mathrm{H}-6)$. Anal. Calcd. for $\mathrm{C}_{15} \mathrm{H}_{13} \mathrm{~N}_{7}$ (291.32): C, 61.84; H, 4.50; N, 33.66. Found: C, 61.75; H, 4.40; N, 33.60.

\section{4-(5-Methyl-1-(p-tolyl)-1H-1,2,3-triazol-4-yl)benzo [4,5] imidazo[1,2-a]pyrimidine (5e)}

Yellow crystals from ethanol, yield (65\%); m.p. 200$202{ }^{\circ} \mathrm{C}$. FT-IR $\left(\mathrm{KBr}, \mathrm{cm}^{-1}\right): 3047,2981(\mathrm{CH}) ; 1635(\mathrm{C}=\mathrm{N})$; $1600(\mathrm{C}=\mathrm{C}) .{ }^{1} \mathrm{H}$ NMR $\left(300 \mathrm{MHz}, \mathrm{CDCl}_{3}\right): \delta=2.49(\mathrm{~s}$, $\left.3 \mathrm{H}, \mathrm{CH}_{3}\right) 2.79$ (s, 3H, $\mathrm{CH}_{3}$ ), 7.26-7.43 (m, 7H, ArH's) 8.43-8.45(d, 1H, ArH), 8.80-8.82 (d 1H, $J=8 \mathrm{~Hz}, \mathrm{ArH})$, 9.65-9.66 (d, $1 \mathrm{H}, J=8 \mathrm{~Hz}$, pyrimidine $\mathrm{H}-6)$. MS (El), m/z (\%): 338 (M-2,65), 323 (35), 304 (50), 275 (90), 262 (70), 249 (20), 221 (30), 132 (100), 91 (90), 77 (20), 65 (40). Anal. Calcd. for $\mathrm{C}_{20} \mathrm{H}_{16} \mathrm{~N}_{6}$ (340.39), C, 70.57; H, 4.74; N, 24.69. Found: C, 70.64; H, 4.48; N, 24.58.

8,10-Dimethyl-4-(5-methyl-1-(p-tolyl)-1H-1,2,3,-triazol-4-yl) pyrido[2',3':3,4]pyrazolo[1,5-a]pyrimidine (5f)

Yellow crystals from ethanol, yield (75\%); m.p. 278$281{ }^{\circ} \mathrm{C}$. FT-IR $\left(\mathrm{KBr}, \mathrm{cm}^{-1}\right)$ : 3064, 2951, $2851(\mathrm{CH}) ; 1624$ 
$(\mathrm{C}=\mathrm{N}) ; 1597(\mathrm{C}=\mathrm{C}) .{ }^{1} \mathrm{H}$ NMR (300 MHz, DMSO-d6): $\delta=2.44\left(\mathrm{~s}, 3 \mathrm{H}, \mathrm{CH}_{3}\right), 2.51\left(\mathrm{~s}, 3 \mathrm{H}, \mathrm{CH}_{3}\right), 2.60(\mathrm{~s}, 3 \mathrm{H}$, $\left.\mathrm{CH}_{3}\right), 2.88\left(\mathrm{~s}, 3 \mathrm{H}, \mathrm{CH}_{3}\right), 6.98-7.00(\mathrm{~s}, 1 \mathrm{H}, J=8 \mathrm{~Hz}$, pyridine H-3), 7.47-7.84 (m, 5H, ArH's) and 8.89-8.87 (d, $1 \mathrm{H}, J=8 \mathrm{~Hz}$, pyrimidine $\mathrm{H}-6) .{ }^{13} \mathrm{C}$ NMR (DMSO- $\mathrm{d}_{6}$ ) $\delta=10.4,19.6,20.6,21.4,101.2,112.4,114.8,122.4,125.7$, 128.6, 130.4, 131.6, 139.4, 141.3, 145.5, 151.3, 153.2, 164.7. Anal. Calcd. for $\mathrm{C}_{21} \mathrm{H}_{19} \mathrm{~N}_{7}$ (369.43), C, 68.28; $\mathrm{H}$, 5.18; N, 26.54. Found: C, 68.20; H, 5.15; N, 26.45.

\section{Synthesis of 5-methly-1-(p-tolyl)-1H-1,2,3-triazol-4-yl) (7-phenylpyrazolo[5,1-c]-[1,2,4]-triazin-3-yl)methanone (10a) and 5-methly-1-(p-tolyl)-1H-1,2,3-triazolo-4-yl)(8-phe- nyl pyrazolo[5.1-c][1,2,4]-triazin-3-yl)methanone (10b)}

Method $A$ Dropwise addition of a solution of the appropriate diazonium salt of heterocyclic amines $(\mathbf{8 a})$ and $(\mathbf{8 b})$ ( $5 \mathrm{mmol}$ ) to a stirred mixture of sodium salt of (2) (1.25 g, $5 \mathrm{mmol})$, sodium acetate $(0.65 \mathrm{~g}, 5 \mathrm{mmol})$ in ethanol $(30 \mathrm{ml})$ at $0-5{ }^{\circ} \mathrm{C}$. The solid so formed after $3 \mathrm{~h}$ and was collected, washed with water and recrystallized to give compound (10a) and, compound (10b), respectively.

Method B A solution of the appropriate diazonium salt of heterocyclic amines (8a) or $(\mathbf{8 b})(5 \mathrm{mmol})$ were added dropwise while stirring a mixture of compound (6) (1.35 g, $5 \mathrm{mmol})$, sodium acetate $(0.65 \mathrm{~g}, 5 \mathrm{mmol})$ in ethanol (30 ml) at $0-5{ }^{\circ} \mathrm{C}$. The resulting solid so formed after $3 \mathrm{~h}$ and was collected, washed with water, and recrystallized to give product identical in all aspects (m.p., mixed m.p. and spectra) with the corresponding compound (10a) and compound (10b), which was obtained in method A.

4-(5-Methyl-1-(p-tolyl)-1H-1,2,3-triazol-4-yl)-7-phenylpyrazol o[5, 1-c][1,2,4]triazine (10a)

Brown crystals from ethanol, yield (75\%); m.p. 215$217^{\circ} \mathrm{C}$. FT-IR (KBr, cm $\left.{ }^{-1}\right)$ : 3058, 2969, $2922(\mathrm{CH}) ; 1681$ (CO); $1639(\mathrm{C}=\mathrm{N}) ; 1544(\mathrm{C}=\mathrm{C}) .{ }^{1} \mathrm{H}$ NMR $(300 \mathrm{MHz}$, DMSO-d6): $\delta=2.44$ (s, 3H, $\left.\mathrm{CH}_{3}\right), 2.64\left(\mathrm{~s}, 3 \mathrm{H}, \mathrm{CH}_{3}\right), 6.33$ (s, 1H, pyrazole H-4), 7.32-7.34 (d, 2H, $J=8 \mathrm{~Hz}$, ArH's), 7.49-7.61 (m, 5H, ArH's), 7.87-7.89 (d, 2H, $J=8 \mathrm{~Hz}$, ArH's) and 9.8 (s, $1 \mathrm{H}$, triazine $\mathrm{H}-4) .{ }^{13} \mathrm{C}$ NMR in DMSOd6 $\delta=10.4,20.6,101.1,120.3,121.4,127.4,128.5,129.5$, 130.2, 134.2, 134.6, 139.6, 142.4, 146.7, 153.1, 154.2. Anal. Calcd. for $\mathrm{C}_{22} \mathrm{H}_{17} \mathrm{~N}_{7} \mathrm{O}$ (395.43): C, 66.82; H, 4.33; N, 24.80. Found: C, 66.89; H, 4.40; N, 24.75.

4-(5-Methyl-1-(p-tolyl)-1H-1,2,3-triazol-4-yl)-8-phenylpyrazol o[5, 1-c][1,2,4]triazine (10b)

Pale brown crystals from ethanol, yield (70\%); m.p. 258$260{ }^{\circ} \mathrm{C}$. FT-IR $\left(\mathrm{KBr}, \mathrm{cm}^{-1}\right)$ : 3046,2919 (CH); 1675 (CO); $1646(\mathrm{C}=\mathrm{N}) ; 1609(\mathrm{C}=\mathrm{C}){ }^{1} \mathrm{H}$ NMR (300 MHz, DMSOd6): $\delta=2.46\left(\mathrm{~s}, 3 \mathrm{H}, \mathrm{CH}_{3}\right), 2.64\left(\mathrm{~s}, 3 \mathrm{H}, \mathrm{CH}_{3}\right), 7.42-7.61$ (m, 7H, ArH's), 8.34-8.37 (d, 2H, J=8 Hz, ArH,s), 9.24 (s, 1H, pyrazole $\mathrm{H}-3)$ and $10.19(\mathrm{~s}, 1 \mathrm{H}$, triazine $\mathrm{H}-4)$. ${ }^{13} \mathrm{C}-\mathrm{NMR}\left(\mathrm{DMSO}-\mathrm{d}_{6}\right.$ ) $\delta=10.4,20.6,102.3,120.6,121.3$, 125.6, 126.8, 126.2,1 29.4, 130.2, 133.4, 134.8, 139.6, 142.5, 1146.7, 151.7, 154.8. Anal. Calcd. for $\mathrm{C}_{22} \mathrm{H}_{17} \mathrm{~N}_{7} \mathrm{O}$ (395.43): C, 66.82; H, 4.33; N, 24.80. Found: C, 66.90; H, 4.37; N, 24.75.

\section{Synthesis of 3-(5-methyl-1-(p-tolyl)-1H-1,2,3-tria- zol-4-y1)-3-oxo-2-(2-phenylhydrazono)propanal (12a) and 3-(5-methyl-1-(p-tolyl)-1H-1,2,3-tria- zol-4-y1)-3-oxo-2-(2-p-tolylhydrazono)propanal (12b)} Method A Dropwise addition of a solution of the appropriate arenediazonium chloride (aniline and $p$-methylaniline) $(5 \mathrm{mmol})$ to a stirred mixture of (2) $(1.25 \mathrm{~g}, 5 \mathrm{mmol})$, sodium acetate $(0.65 \mathrm{~g}, 5 \mathrm{mmol})$ in ethanol $(30 \mathrm{ml})$ at $0-5{ }^{\circ} \mathrm{C}$ the solid so formed after $3 \mathrm{~h}$ and was collected and crystallized from ethanol to afford (12a) and (12b).

Method B Dropwise addition of a solution of the appropriate arenediazonium chloride (aniline and $p$-methylaniline) (5 mmol) to a stirred mixture of (6) (1.35 g, $5 \mathrm{mmol})$, sodium acetate $(0.65 \mathrm{~g}, 5 \mathrm{mmol})$ in ethanol $(30 \mathrm{ml})$ at $0-5{ }^{\circ} \mathrm{C}$. The solid so formed after $3 \mathrm{~h}$ then it was collected and crystallized from ethanol to give products identical in all aspects (m.p., mixed m.p., spectra) with corresponding compounds obtained from method A.

\section{3-(5-Methyl-1-(p-tolyl)-1H-1,2,3-triazol-4-y1)-3-oxo-2-(2-phe- nylhydrazono)propanal (12a)}

Brown crystals from ethanol, yield (85\%); m.p. 215$217{ }^{\circ} \mathrm{C}$. FT-IR (KBr, cm $\left.{ }^{-1}\right): 3435(\mathrm{NH}) ; 2924(\mathrm{CH}) ; 1644$ $(\mathrm{C}=\mathrm{N}),{ }^{1} \mathrm{H}$ NMR $(300 \mathrm{MHz}, \mathrm{DMSO}-\mathrm{d} 6): \delta=2.06(\mathrm{~s}, 3 \mathrm{H}$, $\mathrm{CH}_{3}$ ), 2.34 (s, 3H, $\mathrm{CH}_{3}$ ), 7.26-8.20 (m, 9H, ArH's), 9.75 (s, 1H. CHO) and 14.39 (s, br.,1H, NH). Anal. Calcd. for $\mathrm{C}_{19} \mathrm{H}_{17} \mathrm{~N}_{5} \mathrm{O}_{2}$ (347.38): C, 65.69; H, 4.93; N, 20.16. Found: C, 65.73; H, 4.84; N, 20.12 .

\section{3-(5-Methyl-1-(p-tolyl)-1H-1,2,3-tria-} zol-4-y1)-3-oxo-2-(2-p-tolyl)-hydrazono)propanal (12b)

Dark pink crystals from ethanol, yield (85\%); m.p. 210$212{ }^{\circ} \mathrm{C}$. FT-IR $\left(\mathrm{KBr}, \mathrm{cm}^{-1}\right): 3438(\mathrm{NH}) ; 2922(\mathrm{CH}), 1643$ $(\mathrm{C}=\mathrm{C}),{ }^{1} \mathrm{H}$ NMR $(300 \mathrm{MHz}, \mathrm{DMSO}-\mathrm{d} 6): \delta=2.43(\mathrm{~s}, 3 \mathrm{H}$, $\left.\mathrm{CH}_{3}\right), 2.53\left(\mathrm{~s}, 3 \mathrm{H}, \mathrm{CH}_{3}\right), 2.66\left(\mathrm{~s}, 3 \mathrm{H}, \mathrm{CH}_{3}\right), 7.30-7.72(\mathrm{~m}$, 8H, ArH's), 10.80 (s, 1H, CHO) and 13.9 (s, br., 1H, NH). Anal. Calcd. for $\mathrm{C}_{20} \mathrm{H}_{19} \mathrm{~N}_{5} \mathrm{O}_{2}$ (361.41): C, 66.4; H, 5.30; N, 19.38. Found: C, 66.52; H, 5.38; N, 19.46 .

\section{Synthesis of 2-mercapto-6-(5-methyl-1-(p-tolyl)-1H-1,2,3 -triazol-4-yl)nicotinonitrile (14)}

Method A A mixture of sodium salt (2) (1.25 g, $5 \mathrm{mmol})$ and 2-cyanothioacetamide $(0.5 \mathrm{~g}, 5 \mathrm{mmol})$ in piperidine acetate [piperidine $(2.5 \mathrm{ml})$, water $(5 \mathrm{ml})$ and acetic acid $(2 \mathrm{ml})$ ] was heated under reflux for $15 \mathrm{~min}$, acetic acid 
(1.5 ml) was added to the reaction mixture while boiling then the mixture was cooled and the resulting solid was collected and recrystallized from the proper solvent to give compound (14).

Method B A mixture of (6) (1.35 g, $5 \mathrm{mmol})$ and cyanothioacetamide $(0.5 \mathrm{~g}, 5 \mathrm{mmol})$ in ethanol $(20 \mathrm{ml})$ and a catalytic amount of piperidine $(10 \mathrm{ml})$ was heated under reflux for $4 \mathrm{~h}$. After cooling, the resulting solid was collected and recrystallized from ethanol to afford compound 14 as brown crystals from ethanol, yield (65\%); m.p. $262-265{ }^{\circ} \mathrm{C}$. FT-IR $\left(\mathrm{KBr}, \mathrm{cm}^{-1}\right)$ : 3074, $2962(\mathrm{CH})$; $2218(\mathrm{CN}) ; 1573(\mathrm{C}=\mathrm{C}) .{ }^{1} \mathrm{H}$ NMR $(300 \mathrm{MHz}$, DMSOd6): $\delta=2.43\left(\mathrm{~s}, 3 \mathrm{H}, \mathrm{CH}_{3}\right), 2.61\left(\mathrm{~s}, 3 \mathrm{H}, \mathrm{CH}_{3}\right), 5.87(\mathrm{~s}, 1 \mathrm{H}$, $\mathrm{SH}), 7.34-7.36$ (d, $2 \mathrm{H}, J=8 \mathrm{~Hz}, A$ ArH's), 7.52-7.54 (d, $2 \mathrm{H}, J=8 \mathrm{~Hz}$, ArH's), 7.72-7.74 (d, $1 \mathrm{H}, J=8 \mathrm{~Hz}$, ArH's), 8.39-8.41 (d, $1 \mathrm{H}, J=8 \mathrm{~Hz}$, ArH's). ${ }^{13} \mathrm{C}$ NMR (DMSO-d ${ }_{6}$ ) $\delta=10.4,20.6,104.6,116.5,123.4,125.8,128.4,139.7$, 140.9, 143.8, 144.2, 147.2, 170.8, 173.8. MS (El, m/z (\%): $308(\mathrm{M}+1,20), 294$ (80), 278 (9), 264 (50), 237 (20), 219 (5), 177 (10), 144 (40), 132 (20), 91 (45), 80 (30), 64 (100). Anal. Calcd. for $\mathrm{C}_{16} \mathrm{H}_{13} \mathrm{~N}_{5} \mathrm{O}$ (307.38), C, 62.52; $\mathrm{H}, 4.26$; N, 22.78. Found: C, 62.57; H, 4.23; N, 22.85 .

\section{Synthesis of ethyl 3-amino-6-(5-methyl-1-(p-tolyl)-1H-1,2, 3,-triazol-4-yl)thieno[2,3-b]pyridine-2-carboxylate (15a), 1-(3-amino-6-(5-methyl-1-(p-tolyl)-1H-1,2,3-triazol-4-y1) thino[2,3-b]pyridin-2-yl)-ethan-1-one (15b), 6-(3-amino- 6-(5-methyl-1-(p-tolyl)-1H-1,2,3-triazal-4-yl)thieno[2,3-b] pyridin-2-yl)-(phenyl)methanone (15c), and 3-amino-6-(5- methyl-1-(p-tolyl)-1H-1,2,3-triazol-4-yl)thieno[2,3,-b]-pyri- dine-2-carbonitrile (16)}

A mixture of compound (14) $(2.1 \mathrm{~g}, 5 \mathrm{mmol})$, potassium hydroxide $(0.28 \mathrm{~g}, 5 \mathrm{mmol})$ in $\mathrm{N}, \mathrm{N}$-dimethylformamide $(10 \mathrm{ml})$ was stirred for $2 \mathrm{~h}$ then, the appropriate of ethyl chloroacetate, chloroacetone, $\omega$-bromoacetophenone and chloroacetonitrile $(5 \mathrm{mmol})$ was added while stirring. Stirring was continued for $2 \mathrm{~h}$, the resulting solid was collected and crystallized from the proper solvent to afford compounds $(\mathbf{1 5 a}-\mathbf{c})$, and (16) respectively.

\section{Ethyl 3-amino-6-(5-methyl-1-(p-tolyl)-1H-1,2,3-triazol-4-yl) thieno[2,3-b]pyridine-2-carboxylate (15a)}

Gray crystals from acetic acid, yield (65\%); m.p. $>300{ }^{\circ} \mathrm{C}$. FT-IR $\left(\mathrm{KBr}, \mathrm{cm}^{-1}\right)$ : 3460, $3355\left(\mathrm{NH}_{2}\right)$; 3062, $2970(\mathrm{CH})$, 1666 (CO); $1604(\mathrm{C}=\mathrm{C}) .{ }^{1} \mathrm{H}$ NMR (300 MHz, DMSO-d6): $\delta=1.26\left(\mathrm{t}, 3 \mathrm{H}, J=7 \mathrm{~Hz}, \mathrm{CH}_{2} \mathrm{CH}_{3}\right), 2.34\left(\mathrm{~s}, 3 \mathrm{H}, \mathrm{CH}_{3}\right), 2.64$ (s, $3 \mathrm{H}, \mathrm{CH}_{3}$ ), 4.23 (q, $2 \mathrm{H}, J=7 \mathrm{~Hz}, \mathrm{CH}_{2} \mathrm{CH}_{3}$ ), 6.80 (s, br., $\left.2 \mathrm{H}, \mathrm{NH}_{2}\right), 7.32-7.34(\mathrm{~d}, 2 \mathrm{H}, J=8 \mathrm{~Hz}, \mathrm{ArH}$ 's), 7.52-7.54 (d, $2 \mathrm{H}, J=8 \mathrm{~Hz}, \mathrm{ArH}$ 's), 7.61-7.62 (d, $1 \mathrm{H}, J=8 \mathrm{~Hz}, \mathrm{ArH}$ 's), and 8.81-8.83 (d, $1 \mathrm{H}, J=8 \mathrm{~Hz}$, ArH); ${ }^{13} \mathrm{C}$ NMR (DMSO- $\mathrm{d}_{6}$ ) $\delta=10.4,14.7,20.6,59.5,105.7,121.2,123.2,128.6,133.8$, 139.8, 140.7, 143.8, 44.2, 144.3, 149.7, 155.4, 166.1 Anal. Calcd. for $\mathrm{C}_{20} \mathrm{H}_{19} \mathrm{~N}_{5} \mathrm{O}_{2} \mathrm{~S}$ (393.47): C, 61.05; H, 4.87; N, 17.80 S, 8.1. Found: C, 61.15; H, 4.81; N, 17.76; S, 8.09.

\section{1-(3-Amino-6-(5-methyl-1-(p-tolyl)-1H-1,2,3-triazol-4-yl)} thieno[2,3-b]pyridin-2-yl)ethanone (15b)

Brown crystals from acetic acid, yield (65\%); m.p. 278$280{ }^{\circ} \mathrm{C}$. FT-IR $\left(\mathrm{KBr}, \mathrm{cm}^{-1}\right)$ : 3419, $3321\left(\mathrm{NH}_{2}\right) ; 3092,2920$ $(\mathrm{CH}) ; 1675(\mathrm{CO}) ; 1593(\mathrm{C}=\mathrm{C}) .{ }^{1} \mathrm{H}$ NMR $(300 \mathrm{MHz}$, DMSO-d6), $\delta=2.35\left(\mathrm{~s}, 3 \mathrm{H}, \mathrm{CH}_{3}\right), 2.49\left(\mathrm{~s}, 3 \mathrm{H}, \mathrm{CH}_{3}\right)$, 2.62 (s, $\left.3 \mathrm{H}, \mathrm{CH}_{3}\right), 5.79$ (s, br., $\left.2 \mathrm{H}, \mathrm{NH}_{2}\right), 7.32-7.34(\mathrm{~d}$, $2 \mathrm{H}, J=8 \mathrm{~Hz}$, ArH's), 7.52-7.54 (d, $2 \mathrm{H}, J=8 \mathrm{~Hz}$, ArH's), $7.70-7.72(\mathrm{~d}, 1 \mathrm{H}, J=8 \mathrm{~Hz}, A r H$ 's) and $8.71-8.73(\mathrm{~d}, 1 \mathrm{H}$, $J=8 \mathrm{~Hz}, \operatorname{ArH}$ ); ${ }^{13} \mathrm{C}$ NMR (DMSO-d ${ }_{6}$ ) $\delta=10.4,20.6$, $128.8,120.4,122.7,123.6,134.0,139.8,140.7,143.5$, 144.2, 149.4, 156.1, 190.9. Anal. Calcd. for $\mathrm{C}_{19} \mathrm{H}_{17} \mathrm{~N}_{5} \mathrm{OS}$ (363.45): C, 62.79; H, 4.71; N, 19.27 S, 8.83. Found: C, $62.81 ; \mathrm{H}, 4.71 ; \mathrm{N}, 19.17 ; \mathrm{S}, 8.75$.

\section{(3-Amino-6-(5-methyl-1-(p-tolyl)-1H-1,2,3-triazol-4-yl) thieno[2,3-b]pyridin-2-yl)(phenyl)methanone (15c)}

Brown crystals from acetic acid, yield (65\%); m.p. $220{ }^{\circ} \mathrm{C}$. FT-IR $\left(\mathrm{KBr}, \mathrm{cm}^{-1}\right)$ : 3402, $3286\left(\mathrm{NH}_{2}\right)$; 3066, $2920(\mathrm{CH})$; 1665 (CO); $1608(\mathrm{C}=\mathrm{C}) .{ }^{1} \mathrm{H}$ NMR (300 MHz, DMSOd6): $\delta=2.43\left(\mathrm{~s}, 3 \mathrm{H}, \mathrm{CH}_{3}\right), 2.57\left(\mathrm{~s}, 3 \mathrm{H}, \mathrm{CH}_{3}\right), 5.82$ (s, br., $\left.2 \mathrm{H}, \mathrm{NH}_{2}\right), 7.10-7.87(\mathrm{~m}, 11 \mathrm{H}$, ArH's). Anal. Calcd. for $\mathrm{C}_{24} \mathrm{H}_{19} \mathrm{~N}_{5} \mathrm{OS}$ (425.52), C, 67.74; H, 4.56; N, 16.46; S, 7.54. Found: C, 67.81; H, 4.60; N, 16.53; S, 7.62.

\section{3-Amino-6-(5-methyl-1-(p-tolyl)-1H-1,2,3,-triazol-4-yl) thieno[2,3,-b]pyridine-2-carbonitrile (16)}

Brown crystals from acetic acid, yield (60\%); m.p. $245^{\circ} \mathrm{C}$. FT-IR $\left(\mathrm{KBr}, \mathrm{cm}^{-1}\right)$ : 3344, $3236\left(\mathrm{NH}_{2}\right)$; 3058, 2923 $(\mathrm{CH}) ; 2194(\mathrm{CN}) ; 1639(\mathrm{C}=\mathrm{N}) ; 1581(\mathrm{C}=\mathrm{C}) .{ }^{1} \mathrm{H}$ NMR (300 MHz, DMSO-d6): $\delta=2.43\left(\mathrm{~s}, 3 \mathrm{H}, \mathrm{CH}_{3}\right), 2.57(\mathrm{~s}$, $\left.3 \mathrm{H}, \mathrm{CH}_{3}\right), 7.10-7.87\left(\mathrm{~m}, 7 \mathrm{H}, \mathrm{ArH}\right.$ 's and $\left.\mathrm{NH}_{2}\right), 9.21-9.23$ $(\mathrm{d}, 1 \mathrm{H}, J=8 \mathrm{~Hz}, \mathrm{ArH}) .{ }^{13} \mathrm{C}$ NMR (DMSO-d $\left.{ }_{6}\right) \delta=10.4$, 20.6, 93.8, 115.9, 118.6, 121.7, 125.1, 126.3, 126.7, 130.2, 133.2, 133.9, 138.7, 142.9, 147.9, 156.6. Anal. Calcd. for $\mathrm{C}_{18} \mathrm{H}_{14} \mathrm{~N}_{6} \mathrm{~S}$ (346.42), C, 62.41; H, 4.07; N, 24.26 S, 9.26. Found: C, 62.50; H, 4.17; N, 24.30; S, 9.36.

\section{Synthesis of pyridine derivatives (17), (18) and (20-22)}

A mixture of the appropriate ethyl acetoacetate, acetylacetone, ethyl cyanoacetate, benzoylacetonitrile, malononitrile ( $5 \mathrm{mmol}),(6)(1.35 \mathrm{~g}, 5 \mathrm{mmol})$ and ammonium acetate $(0.37 \mathrm{~g}, 5 \mathrm{mmol})$ in acetic acid $(30 \mathrm{ml})$ was refluxed for $4 \mathrm{~h}$, the resulting solid was collected and recrystallized from the proper solvent to give (17), (18), and (20-22), respectively. 
Ethyl 2-methyl-6-(5-methyl-1-(p-tolyl)-1H-1,2,3,-triazol-4-yl) pyridine-3-carboxylate (17)

White crystals from ethanol, yield (75\%); m.p. 190$192{ }^{\circ} \mathrm{C}$. FT-IR (KBr, cm$\left.{ }^{-1}\right)$ : 3039, 2920, $2800(\mathrm{CH}) ; 1774$ (CO); $1647(\mathrm{C}=\mathrm{N}) ; 1595(\mathrm{C}=\mathrm{C}) .{ }^{1} \mathrm{H}$ NMR $(300 \mathrm{MHz}$, $\left.\mathrm{CDCl}_{3}\right): \delta=1.35\left(\mathrm{t}, 3 \mathrm{H}, J=7 \mathrm{~Hz}, \mathrm{CH}_{2} \mathrm{CH}_{3}\right) 2.48(\mathrm{~s}, 3 \mathrm{H}$, $\left.\mathrm{CH}_{3}\right), 2.57$ (s, 3H, $\left.\mathrm{CH}_{3}\right), 2.79\left(\mathrm{~s}, 3 \mathrm{H}, \mathrm{CH}_{3}\right), 4.22$ (q, $2 \mathrm{H}$, $\left.J=7 \mathrm{~Hz}, \mathrm{CH}_{2} \mathrm{CH}_{3}\right), 7.31-7.33$ (d, $2 \mathrm{H}, J=8 \mathrm{~Hz}$, ArH's), 7.52-7.54 (d, $2 \mathrm{H}, J=8 \mathrm{~Hz}$, ArH's), 8.01-8.03 (d, 1H, $J=8 \mathrm{~Hz}$, ArH's), 8.45-8.47 (d, $1 \mathrm{H}, J=8 \mathrm{~Hz}, \mathrm{ArH}$ 's). ${ }^{13} \mathrm{C}$ NMR (DMSO-d ${ }_{6}$ ) $\delta=10.4,14.4,20.6,25.5,61.8,121.5$, $124.9,126.4,130.1,133.2,133.6,134.7,138.6,143.1$, 149.6, 158.7, 166.5. Anal. Calcd. for $\mathrm{C}_{19} \mathrm{H}_{20} \mathrm{~N}_{4} \mathrm{O}_{2}$ (336.40): C, 67.84; H, 5.99; N, 16.66. Found: C, 67.90; H, 5.85; N, 16.56 .

\section{1-(2-Methyl-6-(5-methyl-1-(p-tolyl)-1H-1,2,3-triazol-4-yl) pyridin-3yl)ethanone (18)}

White crystals from benzene, yield (70\%); m.p. 182$184{ }^{\circ} \mathrm{C}$. FT-IR (KBr, cm $\left.{ }^{-1}\right)$ : 2947, $2924(\mathrm{CH}) ; 1680(\mathrm{CO})$; $1543(\mathrm{CH}) .{ }^{1} \mathrm{H}$ NMR $\left(300 \mathrm{MHz}, \mathrm{CDCl}_{3}\right): \delta=2.48(\mathrm{~s}, 3 \mathrm{H}$, $\left.\mathrm{CH}_{3}\right), 2.63\left(\mathrm{~s}, 3 \mathrm{H}, \mathrm{CH}_{3}\right), 2.79\left(\mathrm{~s}, 3 \mathrm{H}, \mathrm{CH}_{3}\right), 2.82(\mathrm{~s}, 3 \mathrm{H}$, $\mathrm{CH}_{3}$ ). 7.32-7.34 (d, 2H, $\left.J=8 \mathrm{~Hz}, A r H ' s\right), 7.52-7.54$ (d, $2 \mathrm{H}, J=8 \mathrm{~Hz}$, ArH's), 7.86-7.88 (d, $1 \mathrm{H}, J=8 \mathrm{~Hz}$, ArH's), 8.33-8.35 (d, $1 \mathrm{H}, J=8 \mathrm{~Hz}$, ArH's). ${ }^{13} \mathrm{C}-\mathrm{NMR}$ (DMSO- $\mathrm{d}_{6}$ ) $\delta=10.4,20.6$, 25.5, 27.6, 122.4, 125.1, 130.0, 130.5, 133.2, 133.7, 133.8, 138.7, 139.9, 151.2, 157.9, 200.1. MS [El, m/z (\%)]: $306\left(\mathrm{M}^{+}, 30\right), 289$ (20), 278 (100), 263 (40), 220 (30), 205 (5) 160 (50), 144 (60), 117 (30), 91 (60), 77 (20), 65 (55). Anal. Calcd. for $\mathrm{C}_{18} \mathrm{H}_{18} \mathrm{~N}_{4} \mathrm{O}$ (306.37): C, 70.57; $\mathrm{H}$, 5.92; N, 18.29. Found: C, 70.43; H, 5.85; N, 18.35 .

\section{6-(5-Methyl-1-(p-tolyl)-1H-1,2,3-triazol-4-yl)-2-oxo-1,2-di-} hydropyridine-3-carbonitrile (20)

Buff crystals from ethanol, yield (65\%); m.p. $195{ }^{\circ} \mathrm{C}$. FT-IR (KBr, cm $\left.{ }^{-1}\right)$ : 3444 (NH); 3074, 2920, $2858(\mathrm{CH})$; $2225(\mathrm{CN}) ; 1674(\mathrm{CO}) ; 1608(\mathrm{C}=\mathrm{N}) ; 1585(\mathrm{C}=\mathrm{C}) .{ }^{1} \mathrm{H}$ NMR $\left(300 \mathrm{MHz}, \mathrm{CDCl}_{3}\right): \delta=2.48\left(\mathrm{~s}, 3 \mathrm{H}, \mathrm{CH}_{3}\right) 2.70(\mathrm{~s}$, $3 \mathrm{H}, \mathrm{CH}_{3}$ ), 7.09-7.11 (d, 1H, $J=8 \mathrm{~Hz}$, ArH's), 7.19-7.21 (d, $2 \mathrm{H}, J=8 \mathrm{~Hz}$, ArH's), 7.44-7.16 (d, 2H, $J=8 \mathrm{~Hz}$, ArH's), 8.14-8.16 (d, 1H, J=8 Hz, ArH's), 11.65 (s, br., $1 \mathrm{H}, \mathrm{NH})$. Anal. Calcd. for $\mathrm{C}_{16} \mathrm{H}_{13} \mathrm{~N}_{5} \mathrm{O}$ (291.31): C, 65.97; H, 4.50; N, 24.04. Found: C, 65.89; H, 4.59; N, 24.14.

\section{2-Amino-6-(5-methyl-1-(p-tolyl)-1H-1,2,3-triazol-4-yl) pyridine-3-carbonitrile (21)}

White crystals from ethanol, yield (65\%); m.p. $>300{ }^{\circ} \mathrm{C}$. FT-IR (KBr, cm $\left.{ }^{-1}\right)$ : 3421, $3236\left(\mathrm{NH}_{2}\right) ; 2924,2854$ $(\mathrm{CH}) ; 2220(\mathrm{CN}), 1643(\mathrm{C}=\mathrm{O}) ; 1573(\mathrm{C}=\mathrm{C}) .{ }^{1} \mathrm{H}$ NMR $\left(300 \mathrm{MHz}, \mathrm{CDCl}_{3}\right): \delta=2.43\left(\mathrm{~s}, 3 \mathrm{H}, \mathrm{CH}_{3}\right), 2.57(\mathrm{~s}, 3 \mathrm{H}$, $\left.\mathrm{CH}_{3}\right), 6.22\left(\mathrm{~s}, 2 \mathrm{H}, \mathrm{NH}_{2}\right), 7.32-7.34(\mathrm{~d}, 2 \mathrm{H}, J=8 \mathrm{~Hz}$,
ArH's), 7.52-7.54 (d, 2H, $J=8 \mathrm{~Hz}$, ArH's), 8.10-8.12 (d, $1 \mathrm{H}, J=8 \mathrm{~Hz}, \mathrm{ArH}$ 's), $8.56-8.58$ (d, $1 \mathrm{H}, J=8 \mathrm{~Hz}$, ArH's). Anal. Calcd. for $\mathrm{C}_{16} \mathrm{H}_{14} \mathrm{~N}_{6}$ (290.33): C, 66.19; H, 4.86; N, 28.95. Found: C, 66.25; H, 4.75; N, 28.89.

\section{6-(5-Methyl-1-(p-tolyl)-1H-1,2,3-triazol-4-yl)-2-phenylnicoti- nonitrile (22)}

Pale yellow crystals from ethanol, yield (65\%); m.p. 270-273 ${ }^{\circ} \mathrm{C}$. FT-IR (KBr, cm ${ }^{-1}$ ): 3059, 2918 (CH); 2200 $(\mathrm{CN}) ; 1608(\mathrm{C}=\mathrm{C}) .{ }^{1} \mathrm{H}$ NMR $(300 \mathrm{MHz}, \mathrm{DMSO}-\mathrm{d} 6)$ : $\delta=2.42\left(\mathrm{~s}, 3 \mathrm{H}, \mathrm{CH}_{3}\right), 2.62\left(\mathrm{~s}, 3 \mathrm{H}, \mathrm{CH}_{3}\right), 7.32-7.54(\mathrm{~m}$, 9H, Ar's), 7.68-7.88 (d, 1H, $J=8 \mathrm{~Hz}, \mathrm{ArH}), 8.29-8.31$ (d, $1 \mathrm{H}, J=8 \mathrm{~Hz}, \mathrm{ArH})$. Anal. Calcd. for $\mathrm{C}_{22} \mathrm{H}_{17} \mathrm{~N}_{5}$ (351.41): C, 75.19; H, 4.88; N, 19.93. Found: C, 75.16; H, 4.76; N, 19.82 .

\section{Synthesis of 4-(5-methyl-1-(p-tolyl)-1H-1,2,3-triazol-4-yl) thiazol-2-amine (25)}

A mixture of 2-bromo-1-(5-methyl-1-( $p$-tolyl)- $1 H$ 1,2,3-triazol-4-yl)ethanone (23) (2.71 g, $0.01 \mathrm{~mol})$ and thiourea (24) $(0.76 \mathrm{~g}, 0.01 \mathrm{~mol})$ in ethanol $(50 \mathrm{ml})$ was heated under reflux for $30 \mathrm{~min}$. The reaction mixture was poured on ice-cold water and drops of ammonia solution were added. The resulting solid so formed was collected and recrystallized from ethanol gave compound (25) as a white crystal, yield (93\%); m.p. 192-194 ${ }^{\circ} \mathrm{C}$. IR $\left(\mathrm{KBr}, \mathrm{cm}^{-1}\right)$ : 3451, $3231\left(\mathrm{NH}_{2}\right) ;{ }^{1} \mathrm{H}$ NMR $\left(\mathrm{CDCl}_{3}\right)$ : $\delta=2.41\left(\mathrm{~s}, 3 \mathrm{H}, \mathrm{CH}_{3}\right), 2.51\left(\mathrm{~s}, 3 \mathrm{H}, \mathrm{CH}_{3}\right), 6.92-7.50(\mathrm{~m}, 7 \mathrm{H}$, ArH's, $\mathrm{NH}_{2}$ ). ${ }^{13} \mathrm{C}-\mathrm{NMR}$ (DMSO-d 6 ) $\delta=10.4,20.6,119.7$, 125.5, 128.9, 135.4, 139.6, 140.0, 140.7, 142.8, 173.8. MS: $\mathrm{m} / \mathrm{z}=271$ (0.33), 248 (11), 223 (43), 213 (12), 212 (19), 169 (34), 141 (35), 108 (28), 79 (31), 77 (16), 70 (11). Anal. Calcd. For $\mathrm{C}_{13} \mathrm{H}_{13} \mathrm{~N}_{5} \mathrm{~S}$ (271.34): C, 57.54; H, 4.83; N, 25.81; S, 11.82. Found: C, 57.52; H, 4.86; N, 25.79; S, 11.84 .

\section{Synthesis of 4-(5-methyl-1-(p-tolyl)-1H-1,2,3-tria-} zol-4-yl)-5-(aryldiazenyl)thiazol-2-amine (26a,b)

Method A Arenediazonium chloride (5 mmol), which was prepared from aromatic amines $(5 \mathrm{mmol})$, hydrochloric acid $(6 \mathrm{~N}, 6 \mathrm{ml})$, and sodium nitrite $(0.35 \mathrm{~g}, 5 \mathrm{mmol})$, then it was added dropwise with stirring to a cold solution of a mixture of (25) (1.35 g, $5 \mathrm{mmol})$ and sodium acetate trihydrate $(1.3 \mathrm{~g} 10 \mathrm{mmol})$ in ethanol $(50 \mathrm{ml})$. The resulting solid was collected and recrystallized from the proper solvent gave $(\mathbf{2 6 a}, \mathbf{b})$.

Method B A mixture of (28) (2 g, $5 \mathrm{mmol}$ ), thiourea $(0.46 \mathrm{~g}, 6 \mathrm{mmol})$ and triethylamine $(0.5 \mathrm{~g}, 0.72 \mathrm{ml}$, $5 \mathrm{mmol})$ in ethanol $(25 \mathrm{ml})$ was heated under reflux for $2 \mathrm{~h}$. The resulting solid was collected, washed with water, and crystallized from ethanol to give (26a). 
4-(5-Methyl-1-(p-tolyl)-1H-1,2,3-tria-

zol-4-yl)-5-(phenyldiazenyl)thiazol-2-amine (26a)

A yellow crystals from ethanol, yield (65\%); m.p. 218$220{ }^{\circ} \mathrm{C}$. IR $\left(\mathrm{KBr}, \mathrm{cm}^{-1}\right): 3444,3275\left(\mathrm{NH}_{2}\right) ;{ }^{1} \mathrm{H}$ NMR $\left(\mathrm{CDCl}_{3}\right): \delta=2.48\left(\mathrm{~s}, 3 \mathrm{H}, \mathrm{CH}_{3}\right), 2.63\left(\mathrm{~s}, 3 \mathrm{H}, \mathrm{CH}_{3}\right)$, 7.27-7.92 (m, 11H, ArH's, $\left.\mathrm{NH}_{2}\right) .{ }^{13} \mathrm{C}$ NMR (DMSO-d $\left.\mathrm{d}_{6}\right)$ $\delta=10.4,20.6,103.2$, 118.1, 121.6, 127.4, 129.0, 130.2, $134.3,139.8,141.6,141.9,143.8,155.0,176.2$. MS: $\mathrm{m} \backslash \mathrm{z}=335$ (15), 334 (21), 305 (10), 200 (61), 198 (35), 185 (13), 183 (15), 157 (14), 128 (14), 115 (16), 105 (25), 103 (45), 91 (21), 43 (99). Anal. Calcd. for $\mathrm{C}_{19} \mathrm{H}_{17} \mathrm{~N}_{7} \mathrm{~S}$ (375.45): C, 60.78; H, 4.56; N, 26.11; S, 8.54. Found: C, 60.85; H, $4.64 ; \mathrm{N}, 26.21 ; \mathrm{S}, 8.35$.

Synthesis of 5-((4-chlorophenyl)diazenyl)-4-(5-methyl-1-(p-to lyl)-1H-1,2,3-triazol-4-yl)thiazol-2-amine (26b)

Yellow crystals from acetic acid gave, yield (65\%); m.p. 168-170 ${ }^{\circ} \mathrm{C} .{ }^{1} \mathrm{H} \mathrm{NMR}\left(\left(\mathrm{CD}_{3}\right)_{2} \mathrm{SO}\right): \delta=2.43\left(\mathrm{~s}, 3 \mathrm{H}, \mathrm{CH}_{3}\right)$, 2.52 (s, 3H, $\mathrm{CH}_{3}$ ), 7.44-7.68 (m, 8H, ArH's), 8.48 (s, 2H, $\mathrm{NH}_{2}$ ). Anal. Calcd. for $\mathrm{C}_{19} \mathrm{H}_{16} \mathrm{ClN}_{7} \mathrm{~S}$ (409.90): C, 55.67; $\mathrm{H}$, 3.93; N, 23.92; S, 7.82. Found: C, 55.52; H, 3.81; N, 24.10; S, 7.70.

\section{Synthesis of 1-(4-(5-methyl-1-(p-tolyl)-1H-1,2,3-triazol-4-yl) thiazol-2-yl)-3-phenylthiourea (27)}

A mixture of 4-(5-methyl-1-(p-tolyl)- $1 \mathrm{H}$-1,2,3-triazol4-yl)thiazol-2-amine (25) (1.35 g, $5 \mathrm{mmol}$ ), phenyl isothiocyanate $(0.6 \mathrm{ml}, 5 \mathrm{mmol})$ and potassium hydroxide $(0.28 \mathrm{~g}, 5 \mathrm{mmol})$ in DMF $(10 \mathrm{ml})$ was stirred for $3 \mathrm{~h}$. Then the mixture was poured on ice water containing $\mathrm{HCl}$, the resulting solid was collected and crystallized from ethanol and gave white crystals, yield (75\%); m.p. 200-202 ${ }^{\circ} \mathrm{C}$. IR (KBr, cm $\left.{ }^{-1}\right)$ : $3264(\mathrm{NH}), 3220(\mathrm{NH}), 1240(\mathrm{C}=\mathrm{S}) ;{ }^{1} \mathrm{H}$ NMR $\left(\left(\mathrm{CD}_{3}\right)_{2} \mathrm{SO}\right): \delta=2.42\left(\mathrm{~s}, 3 \mathrm{H}, \mathrm{CH}_{3}\right), 2.58(\mathrm{~s}, 3 \mathrm{H}$, $\mathrm{CH}_{3}$ ), 7.20-7.65 (m, 10H, ArH's), 10.95 (s, 1H, NH), 11.92 (s, 1H, NH); Ms: m/z = 406 (4), 390 (13), 370 (14), 297 (10), 284 (51), 271 (42), 252 (11), 242 (49), 210 (11), 200 (52), 183 (23), 168 (28), 156 (15), 144 (36), 125 (15), 115 (51), 105 (19), 102 (15), 91 (99), 85 (27), 77 (52), 69 (78), 65 (100), 52 (23), 45 (52). Anal. Calcd. for $\mathrm{C}_{20} \mathrm{H}_{18} \mathrm{~N}_{6} \mathrm{~S}_{2}$ (406.53): C, 59.09; H, 4.46; N, 20.67; S, 15.78. Found: C, 58.89; H, 4.64; N, 20.75; S, 15.84 .

\section{Synthesis of 2-[5-methyl-(p-tolyl)-1-H-1, 2, 3-tri-}

\section{zol-4-yl]-2-oxo-N-phenylacetohydrazonoyl bromide (28)}

A mixture of (29) (35.6 g, $0.1 \mathrm{~mol}$ ) and $N$-nitrosoacetanilide [35] (10.4 g, $0.1 \mathrm{~mol})$ in ethanol $(100 \mathrm{ml})$ was stirred for $2 \mathrm{~h}$ at room temperature. The resulting solid was collected, washed with water and recrystallized from ethanol gave yellow crystals, yield (60\%); m.p. 174-176 ${ }^{\circ} \mathrm{C}$. IR (KBr, cm $\left.{ }^{-1}\right)$ : $3441(\mathrm{NH}), 1651(\mathrm{C}=\mathrm{O}), 1597(\mathrm{C}=\mathrm{N})$; ${ }^{1} \mathrm{H}$ NMR $\left(\mathrm{CDCl}_{3}\right): \delta=2.48\left(\mathrm{~s}, 3 \mathrm{H}, \mathrm{CH}_{3}\right), 2.59(\mathrm{~s}, 3 \mathrm{H}$, $\mathrm{CH}_{3}$ ), 7.10-7.41 (m, 9H, ArH's), 8.76 (s, 1H, NH); MS: $\mathrm{m} \backslash \mathrm{z}=399$ (22), 397 (22), 362 (18), 360 (55), 358 (56), 281 (25), 279 (50), 90 (18), 62 (15), 43 (99). Anal. Calcd. for $\mathrm{C}_{18} \mathrm{H}_{16} \mathrm{BrN}_{5} \mathrm{O}$ (398.26): C, 54.28; H, 4.05; N, 17.59. Found: C, 54.15; H, 4.14; N, 17.66.

\section{Synthesis of dimethyl(2-(5-methyl-1-(p-tolyl)-1H-1,2,3-tria-} zol-4-yl)-2-oxoethyl)sulfonium bromide (29)

A mixture of (23) $(29.4 \mathrm{~g}, 0.1 \mathrm{~mol})$ with dimethylsulfide $(6.2 \mathrm{~g}, 0.1 \mathrm{~mol})$ in ethanol $(50 \mathrm{ml})$ was refluxed for $30 \mathrm{~min}$. The reaction mixture was cooled to room temperature and then diluted with diethyl ether to complete precipitation. The resulting solid was collected and crystallized from ethanol to give white crystals, yield (78\%); m.p. $134-135^{\circ} \mathrm{C}$.

\section{Synthesis of 1,3,4-thiadiazole (31a-d),}

2-((4-(5-methyl-1-(p-tolyl)-1H-1,2,3-triazol-4-yl)thiazol-2-yl) imino)-3-phenylthiazolidin-4-one (33) and N-(3,4-diphenylthiazol-2(3H)-ylidene)-4-(5-methyl-1-(p-tolyl)-1H-1,2,3 -triazol-4-yl)thiazol-2-amine (32)

A mixture of 4-(5-methyl-1-(p-tolyl)-1H-1,2,3-triazol4-yl)thiazol-2-amine (25) (1.35 g, $5 \mathrm{mmol}$ ), phenyl isothiocyanate $(0.6 \mathrm{ml}, 5 \mathrm{mmol})$ and potassium hydroxide $(0.28 \mathrm{~g}, 5 \mathrm{mmol})$ in DMF $(10 \mathrm{ml})$ was stirred for $3 \mathrm{~h}$. then added appropriate hydrazonoyl chlorides $(\mathbf{3 0 a}-\mathbf{d})$, or ethyl 2-chloroacetate $(0.61 \mathrm{~g}, 5 \mathrm{mmol})$ or 2-bromo1-phenylethanone $(0.99 \mathrm{~g}, 5 \mathrm{mmol})$ and complete stirring $2 \mathrm{~h}$, the resulting solid collected and recrystallized to give (31a-d), (32) and (33), respectively.

\section{Ethyl 5-((4-(5-methyl-1-(p-tolyl)-1H-1,2,3-triazol-4-yl) thiazol-2-yl)imino)-4-phenyl-4,5-dihydro-1,3,4-thiadia- zole-2-carboxylate (31a)}

Yellow crystals from acetic acid, yield (74\%); m.p. 253254 ${ }^{\circ} \mathrm{C}$. IR $\left(\mathrm{KBr}, \mathrm{cm}^{-1}\right): 1725(\mathrm{C}=\mathrm{O}), 1597(\mathrm{C}=\mathrm{N}), 1248$, 1059 (CO); ${ }^{1} \mathrm{H}$ NMR $\left(\mathrm{CDCl}_{3}\right): \delta=1.42(\mathrm{t}, 3 \mathrm{H}, J=7 \mathrm{~Hz}$, $\mathrm{CH}_{2} \mathrm{CH}_{3}$ ), $2.48\left(\mathrm{~s}, 3 \mathrm{H}, \mathrm{CH}_{3}\right), 2.80\left(\mathrm{~s}, 3 \mathrm{H}, \mathrm{CH}_{3}\right), 4.49$ (q, $2 \mathrm{H}, J=7 \mathrm{~Hz}, \mathrm{CH}_{2} \mathrm{CH}_{3}$ ), 7.27-7.59 (m, 9H, ArH's), 8.55 (s, $1 \mathrm{H}$, thiazole $\mathrm{H}-5$ ). ${ }^{13} \mathrm{C}-\mathrm{NMR}$ (DMSO- $\mathrm{d}_{6}$ ) $\delta=10.4,14.5$, 20.6, 62.9, 122.8, 123.7, 125.6, 127.9, 129.0, 130.1, 136.1, 139.6, 141.4, 143.8, 143.9, 148.2, 159.4, 161.1, 171.0. MS: $\mathrm{m} / \mathrm{z}=504$ (10), 503 (37), 475 (58), 344 (32), 343 (15), 292 (16), 200 (100), 186 (24), 168 (33), 161 (23), 157 (13), 144 (22), 135 (11), 115 (20), 91 (72), 77 (47), 65 (23). Anal. Calcd. for $\mathrm{C}_{24} \mathrm{H}_{21} \mathrm{~N}_{7} \mathrm{O}_{2} \mathrm{~S}_{2}$ (503.60) C, 57.24; H, 4.20; N, 19.47; S, 12.73. Found: C, 57.31; H, 4.15; N, 19.57; S, 12.82 .

Ethyl 5-((4-(5-methyl-1-(p-tolyl)-1H-1,2,3-triazol-4-yl) thiazol-2-yl)imino)-4-(p-tolyl)-4,5-dihydro-1,3,4-thiadiazole-2-carboxylate (31b)

Yellow crystals from acetic acid, yield (74\%); m.p. 174-175 ${ }^{\circ} \mathrm{C}$. IR (KBr, cm$\left.{ }^{-1}\right): 1677(\mathrm{C}=\mathrm{O}), 1605(\mathrm{C}=\mathrm{N})$, 
1244,1061 (CO); ${ }^{1} \mathrm{H}$ NMR $\left(\mathrm{CDCl}_{3}\right): \delta=1.36-1.46(\mathrm{t}, 3 \mathrm{H}$, $\left.J=7 \mathrm{~Hz}, \mathrm{CH}_{2} \mathrm{CH}_{3}\right), 2.33\left(\mathrm{~s}, 3 \mathrm{H}, \mathrm{CH}_{3}\right), 2.45\left(\mathrm{~s}, 3 \mathrm{H}, \mathrm{CH}_{3}\right)$, $2.80\left(\mathrm{~s}, 3 \mathrm{H}, \mathrm{CH}_{3}\right), 4.45-4.52\left(\mathrm{q}, 2 \mathrm{H}, J=7 \mathrm{~Hz}, \mathrm{CH}_{2} \mathrm{CH}_{3}\right)$, 7.13-7.57 (m, 8H, ArH's), 8.55 (s, $1 \mathrm{H}$, thiazole $\mathrm{H}-5)$; MS: $\mathrm{m} / \mathrm{z}=517$ (5), 406 (11), 397 (15), 394 (10), 322 (44), 293 (26), 275 (14), 222 (13), 181 (12), 157 (14), 154 (14), 145 (16), 134 (15), 106 (100), 83 (50), 79 (56), 77 (46), 65 (54), 51 (35). Anal. Calcd. for $\mathrm{C}_{25} \mathrm{H}_{23} \mathrm{~N}_{7} \mathrm{O}_{2} \mathrm{~S}_{2}$ (517.63) C, 58.01; H, 4.48; N, 18.94; S, 12.39. Found: C, 58.12; H, 4.58; N, 19.10; S, 12.47 .

\section{1-(5-((4-(5-methyl-1-(p-tolyl)-1H-1,2,3-triazol-4-yl)thia- zol-2-yl)imino)-4-phenyl-4,5-dihydro-1,3,4-thiadiazol-2-yl) ethanone (31c)}

Yellow crystals from acetic acid, yield (74\%); m.p. 215$217{ }^{\circ} \mathrm{C}$. IR $\left(\mathrm{KBr}, \mathrm{cm}^{-1}\right): 1649(\mathrm{C}=\mathrm{O}), 1549(\mathrm{C}=\mathrm{N}) ;{ }^{1} \mathrm{H}$ NMR $\left(\mathrm{CDCl}_{3}\right): \delta=2.55\left(\mathrm{~s}, 9 \mathrm{H}, \mathrm{CH}_{3}\right), 7.12-7.47(\mathrm{~m}, 9 \mathrm{H}$, ArH's), 8.55 (s, $1 \mathrm{H}$, thiazole H-5). ${ }^{13} \mathrm{C}$ NMR (DMSO$\left.\mathrm{d}_{6}\right) \delta=10.4,20.6,24.6,122.8,123.6,125.1,127.7,127.9$, 130.6, 136.1, 140.2, 142.1, 143.7, 147.1, 148.2, 171.1, 189.2. MS: $\mathrm{m} / \mathrm{z}=473$ (7), 435 (15), 429 (12), 423 (12), 418 (14), 409 (11), 370 (94), 342 (46), 314 (25), 299 (12), 295 (13), 286 (17), 279 (17), 272 (30), 239 (12), 205 (17), 180 (13), 171 (35), 149 (30), 144 (38), 142 (30), 134 (54), 132 (13), 116 (38), 106 (35), 98 (22), 91 (100), 83 (44), 69 (42), 67 (33), 57 (52), 55 (80), 51 (32), 43 (44). Anal. Calcd. for $\mathrm{C}_{23} \mathrm{H}_{19} \mathrm{~N}_{7} \mathrm{OS}_{2}$ (473.57) C, 58.33; H, 4.04; N, 20.70; S, 13.54. Found: C, 58.25; H, 3.90; N, 20.56; S, 13.49 .

\section{1-(5-((4-(5-methyl-1-(p-tolyl)-1H-1,2,3-triazol-4-yl)thia- zol-2-yl)imino)-4-(p-tolyl)-4,5-dihydro-1,3,4-thiadiazol-2-yl) ethanone (31d)}

Yellow crystals from acetic acid, yield (74\%); m.p. 211$212{ }^{\circ} \mathrm{C}$. IR $\left(\mathrm{KBr}, \mathrm{cm}^{-1}\right): 1688(\mathrm{C}=\mathrm{O}), 1601(\mathrm{C}=\mathrm{N}) ;{ }^{1} \mathrm{H}$ $\operatorname{NMR}\left(\mathrm{CDCl}_{3}\right): \delta=2.35\left(\mathrm{~s}, 3 \mathrm{H}, \mathrm{CH}_{3}\right), 2.46\left(\mathrm{~s}, 3 \mathrm{H}, \mathrm{CH}_{3}\right)$, $2.66\left(\mathrm{~s}, 3 \mathrm{H}, \mathrm{CH}_{3}\right), 2.81\left(\mathrm{~s}, 3 \mathrm{H}, \mathrm{CH}_{3}\right), 7.16-7.61(\mathrm{~m}, 8 \mathrm{H}$, ArH's), 8.55 (s, $1 \mathrm{H}$, thiazole H-5); MS: $\mathrm{m} / \mathrm{z}=491$ (23), 488 (11), 487 (36), 459 (58), 357 (11), 285 (19), 276 (23), 201 (24), 200 (100), 186 (24), 175 (18), 168 (27), 157 (13), 142 (21), 132 (17), 115 (14), 105 (16), 91 (57), 65 (19). Anal. Calcd. for $\mathrm{C}_{24} \mathrm{H}_{21} \mathrm{~N}_{7} \mathrm{OS}_{2}$ (487.60) C, 59.12; H, 4.34; N, 20.11; S, 13.15. Found: C, 59.21; H, 4.43; N, 20.25; S, 13.22 .

$\mathrm{N}$-(3,4-diphenylthiazol-2(3H)-ylidene)-4-(5-methyl-1-( $p$-tolyl) -1H-1,2,3-triazol-4-yl)thiazol-2-amine (32)

Yellow crystals from acetic acid, yield (72\%); m.p. 270$272{ }^{\circ} \mathrm{C}$. IR $\left(\mathrm{KBr}, \mathrm{cm}^{-1}\right): 3114(=\mathrm{CH}) ;{ }^{1} \mathrm{H}$ NMR $\left(\mathrm{CDCl}_{3}\right)$ : $\delta=2.42\left(\mathrm{~s}, 3 \mathrm{H}, \mathrm{CH}_{3}\right), 2.78\left(\mathrm{~s}, 3 \mathrm{H}, \mathrm{CH}_{3}\right), 6.38(\mathrm{~s}, 1 \mathrm{H}, \mathrm{CH})$, 7.15-7.52 (m, 14H, ArH's), 8.67 (s, 1H, thiazole H-5); MS: $\mathrm{m} / \mathrm{z}=506$ (8), 505 (23), 477 (25), 294 (44), 278 (21), 275
(23), 251 (11), 200 (32), 180 (12), 168 (13), 134 (21), 115 (15), 105 (38), 91 (69), 77 (100), 65 (47), 51 (28), 45 (15). Anal. Calcd. for $\mathrm{C}_{28} \mathrm{H}_{22} \mathrm{~N}_{6} \mathrm{~S}_{2}$ (506.64): C, 66.38; H, 4.38; N, 16.59; S, 12.66. Found: C, 66.27; H, 4.45; N, 16.67; S, 12.72 .

\section{2-((4-(5-Methyl-1-(p-tolyl)-1H-1,2,3-triazol-4-yl)thiazol-2-yl) imino)-3-phenylthiazolidin-4-one (33)}

Pink crystals from acetic acid, yield (78\%); m.p. 285-287 ${ }^{\circ} \mathrm{C}$. IR $\left(\mathrm{KBr}, \mathrm{cm}^{-1}\right)$ : $1730(\mathrm{C}=\mathrm{O}) ;{ }^{1} \mathrm{H}$ NMR $\left(\left(\mathrm{CD}_{3}\right)_{2} \mathrm{SO}\right): \delta=2.43\left(\mathrm{~s}, 3 \mathrm{H}, \mathrm{CH}_{3}\right), 2.67\left(\mathrm{~s}, 3 \mathrm{H}, \mathrm{CH}_{3}\right)$, $3.96\left(\mathrm{~s}, 2 \mathrm{H}, \mathrm{CH}_{2}\right), 7.37-7.77(\mathrm{~m}, 9 \mathrm{H}, \mathrm{ArH}$ 's), 8.67 (s, $1 \mathrm{H}$, thiazole $\mathrm{H}-5) ; \mathrm{MS}: \mathrm{m} / \mathrm{z}=447$ (7), 446 (26), 418 (100), 201 (18), 200 (91), 186 (22), 168 (30), 144 (19), 142 (24), 115 (20), 91 (48), 77 (42), 65 (21); Anal. Calcd. for $\mathrm{C}_{22} \mathrm{H}_{18} \mathrm{~N}_{6} \mathrm{OS}_{2}(446.55) \mathrm{C}, 59.17 ; \mathrm{H}, 4.06 ; \mathrm{N}, 18.82 ; \mathrm{S}$, 14.36. Found: C, 59.17; H, 4.06; N, 18.82; S, 14.36 .

\section{Conclusions}

New series of pyrazolo[1,5-a]pyrimidines, pyrazolo[5,1c] triazines, thieno[2,3-b]pyridines and polysubstituted pyridines containing the 1,2,3,-triazole moiety were synthesized via reactions of sodium 3-(5-methyl-1-( $p$-tolyl)$1 H$-1,2,3-triazol-4-yl)-3-oxoprop-1-en-1-olate with the appropriate heterocyclic amines and its diazonium salt. In addition, 1,3,4-thiadiazoles and, 1,3-thiazoles were acquired in a decent yield via the reaction of substituted thiourea with the appropriate hydrazonoyl chlorides and halogenated ketenes.

\section{Abbreviations \\ COX-2: cyclooxygenase-2; CNS: the central nervous system; HMG-CoA: the enzyme 3-hydroxy-3-methyl-glutaryl-co-enzyme A; KDR: kinase insert domain receptor; PDE: a phosphodiesterase; MW: molecular weight; TLC: thin layer chromatography.}

\section{Authors' contributions}

$A O A, N A A, Y H Z$ : design the research, performed the research, analyzed the data, wrote the paper. All authors read and approved the final manuscript.

\section{Author details}

${ }^{1}$ Department of Chemistry, Faculty of Science, Cairo University, Giza 12613, Egypt. ${ }^{2}$ Department of Chemistry, Faculty of Science, Beni-Suef University, Beni-Suef 62514, Egypt. ${ }^{3}$ Department of Chemistry, Faculty of Science and Humanity Studies at Al-Quwayiyah, Shaqra University, Al-Quwayiyah 11971, Saudi Arabia.

\section{Competing interests}

The authors declare that they have no competing interests.

Consent for publication

All authors consent to the publication.

\section{Publisher's Note}

Springer Nature remains neutral with regard to jurisdictional claims in published maps and institutional affiliations. 
Received: 16 March 2017 Accepted: 31 May 2017

Published online: 12 June 2017

\section{References}

1. Novinson T, Bhooshan B, Okabe T, Revankar GR, Wilson HR, Robins RK, Senga K (1976) Novel heterocyclic nitrofurfural hydrazones. In vivo nosomal activity. J Med Chem 19(4):512-516

2. Senga K, Novinson T, Wilson HR, Robins RK (1981) Synthesis and antischistosomal activity of certain pyrazolo[1,5-a]pyrimidines. J Med Chem 24(5):610-613

3. Suzuki M, Iwasaki H, Fujikawa Y, Sakashita M, Kitahara M, Sakoda R (2001) Synthesis and biological evaluations of condensed pyridine and condensed pyrimidine-based HMG-CoA reductase inhibitors. Bioorg Med Chem Lett 11(10):1285-1288

4. Almansa C, Merlos M, Rafanell JG, Arriba AF, Cavalcanti FL, Gomez LA, Miralles A, Forn J (2001) Synthesis and SAR of a new series of COX-2-selective inhibitors: pyrazolo[1,5-a]pyrimidines. J Med Chem 44(3):350-361

5. Fraley ME, Hoffman WF, Rubino RS, Hungate RW, Tebben AJ, Rutledge RZ, McFall RC, Huckle WR, Kendall RL, Coll KE, Thomas KA (2002) Synthesis and initial SAR studies of 3, 6-disubstituted pyrazolo[1,5-a] pyrimidines: a new class of KDR kinase inhibitors. Bioorg Med Chem Lett 12(19):2767-2770

6. Novinson T, Hanson R, Dimmitt MK, Simmon LN, Robins RK, O'Brien DE (1974) 3-Substituted 5,7-dimethylpyrazolo[1,5-a]pyrimidines, 3',5'-cyclic AMP phosphodiesterase inhibitors. J Med Chem 17(6):645-648

7. Selleri S, Bruni F, Costagli C, Costanzo A, Guerrini G, Ciciani G, Costa B, Martini C (2001) 2-Arylpyrazolo[1,5-a]pyrimidin-3-yl acetamides. New potent and selective peripheral benzodiazepine receptor ligands. Bioorg Med Chem 9(10):2661-2671

8. Zaki YH, Sayed AR, Elroby SA (2016) Regioselectivity of 1,3-dipolar cycloadditions and antimicrobial activity of isoxazoline, pyrrolo[3,4-d] isoxazole-4,6-diones, pyrazolo[3,4-d]pyridazines and pyrazolo[1,5-a] pyrimidines. Chem Central J 10(17):1-13

9. Kirkipatrick WE, Okabe T, Hillyard IW, Robins RK, Novinson T, Dren AT (1977) 3-Halo-5,7-dimethylpyrazolo[1,5-a]pyrimidines, a nonbenzodiazepinoid class of antianxiety agents devoid of potentiation of central nervous system depressant effects of ethanol or barbiturates. J Med Chem 20(3):386-393

10. Martins MAP, Scapin E, Frizzo CP, Rosa FA, Bonacorso HG, Zanatta N (2009) 2-Methyl-7-substituted pyrazolo[1,5-a]pyrimidines: highly regioselective synthesis and bromination. J Braz Chem Soc 20(2):205-213

11. Rusinov VL, Ulomskii EN, Chupakhin ON, Charushin VN (2008) Azolo[5,1-c]-1,2,4-triazines as a new class of antiviral compounds. Russ Chem Bull Int Ed 57(5):985-1014

12. Arden GM, Grant DJW, Partridge MW (1970) Action of tumour inhibitory pyrazolotriazines on Klebsiella aerogenes - II: inhibition by 6-halogenoacetyl-3-methyl-4-methylenepyrazolo[3,2-c]-as-triazine and its antagonism. Biochem Pharmacol 19(1):71-89

13. Ledenyovaa IV, Didenkoa W, Dotsenko W, Shikhalieva KS (2014) Azo-coupling of pyrazole-3(5)-diazonium chlorides with cyanothioacetamide: a convenient synthesis of pyrazolo[5,1-c][1,2,4] triazine-3-carbothioamides. Tetrahedron Lett 55(6):1239-1242

14. Guerrini G, Ciciani G, Bruni F, Selleri S, Guarino C, Melani F, Montali M, Daniele S, Martini C, Ghelardini C, Norcini M, Ciattini S, Costanzo A (2010) New fluoro derivatives of the pyrazolo[5,1-c] $[1,2,4]$ benzotriazine 5-oxide system: evaluation of fluorine binding properties in the benzodiazepine site on $\gamma$-aminobutyric acid type a (GABAA) receptor. Design, synthesis, biological, and molecular modeling investigation. J Med Chem 53(21):7532-7548

15. Guerrini G, Ciciani G, Bruni F, Selleri S, Melani F, Daniele S, Martini C, Costanzo A (2011) New 3-, 8-disubstituted pyrazolo[5,1-c][1,2,4]benzotriazines useful for studying the interaction with the $\mathrm{HBP}-3$ area (hydrogen bond point area) in the benzodiazepine site on the $\gamma$-aminobutyric acid type A (GABAA) receptor. Bioorg Med Chem 19(10):3074-3085
16. Cornnello M, Ciciani G, Mini E, Guerrini G, Caciagli B, Selleri S, Costanzo A, Mazzei T (2005) Cytotoxic activity of 3-nitropyrazolo[5,1-c][1,2,4]-benzotriazine derivatives: a new series of anti-proliferative agents. Anticancer Drugs 16(6):645-661

17. Ciciani G, Coronnello M, Guerrini G, Selleri S, Cantore M, Failli P, Mini E, Costanzo A (2008) Synthesis of new pyrazolo[5,1-c][1,2,4]benzotriazines, pyrazolo[5,1-c]pyrido[4,3-e][1,2,4]triazines and their open analogues as cytotoxic agents in normoxic and hypoxic conditions. Bioorg Med Chem 16(21):9409-9419

18. Zhang LJ, Yang MY, Sun ZH, Tan CX, Weng JQ, Wu HK, Liu XH (2014) Synthesis and antifungal activity of 1,3,4-thiadiazole derivatives containing pyridine group. Lett Drug Des Discov 11(9):1107-1111

19. Yan SL, Yang MY, Sun ZH, Min LJ, Tan CX, Weng JQ, Wu HK, Liu XH (2014) Synthesis and antifungal activity of 1,2,3-thiadiazole derivatives containing 1,3,4-thiadiazole moiety. Lett Drug Des Discov 11(7):940-943

20. Tong JY, Sun NB, Wu HK, Liu XH (2013) Synthesis, crystal structure and biological activity of $N$-(5-(O-tolyl)-1,3,4-thiadiazol-2-yl)cyclopropanecarboxamide. J Chem Soc Pak 35(5):1349-1353

21. Yang MY, Zhao W, Sun ZH, Tan CX, Weng JQ, Liu XH (2015) Synthesis and biological activity of acylthiourea derivatives contain 1,2,3-thiadiazole and 1,3,4-thiadiazole. Lett Drug Des Discov 12(4):314-318

22. Li Z, Wang X, Da Y (2001) Synthesis of 2-(5-(2-chlorophenyl)2-furoylamino)-5-aryloxymethyl-1,3,4-thiadiazoles under microwave irradiation. Synth Commun 31(12):1829-1836

23. Liu X, Shi Y, Ma Y, Zhang C, Dong W, Pan L, Wang B, Li Z (2009) Synthesis, antifungal activities and 3D-QSAR study of $\mathrm{N}$-(5-substituted-1,3,4-thiadiazol-2-yl)cyclopropane carboxamides. Eur J Med Chem 44(7):2782-2786

24. Ahmad T, Singh AK, Jaiswal N, Singh D (2012) Synthesis and pharmacological activity of 1,3,4-thiadiazole derivatives: a review. Int Res J Pharm 3(3):70-82

25. Gomha SM, Khalil KD, El-Zanate AM, Riyadh SM (2013) A facile green synthesis and anti-cancer activity of bis-arylhydrazononitriles, triazolo[5,1c] $[1,2,4]$ triazine, and 1,3,4-thiadiazoline. Heterocycles 87(5):1109-1120

26. Gomha SM, Riyadh SM (2011) Synthesis under microwave irradiation of $[1,2,4]$ triazolo[3,4- $b][1,3,4]$ thiadiazoles and other diazoles bearing indole moieties and their antimicrobial evaluation. Molecules 16(10):8244-8256

27. Gomha SM, Abdel-Aziz HA (2012) Synthesis of new heterocycles derived from 3-(3-methyl-1Hindol-2-yl)-3-oxopropanenitrile as potent antifungal agents. Bull Korean Chem Soc 33(9):2985-2990

28. Ahmed SA, Hussein AM, Hozayen WGM, El-Ghandour AHH, Abdelhamid AO (2007) Synthesis of some pyrazolopyrimidines as purine analogues. J Heterocycl Chem 44(4):803-810

29. Ahmed SA, Ahmed OM, Abdelhamid AO (2014) Synthesis and anti-tumor activities of new $[1,2,4]$ triazolo[1,5-a]pyrimidine derivatives. Eur J Chem 5(2):334-338

30. Abdelhamid AO, El-Idreesy TT, Abdelriheem NA, Dawoud HRM (2016) Green one-pot solvent-free synthesis of pyrazolo[1,5-a]pyrimidines, azolo[3,4-d]pyridiazines, and thieno[2,3-b]pyridines containing triazole moiety. J Heterocycl Chem 53(3):710-718

31. Cao Z-P, Dong W-J, Dong H-S (2009) One pot synthesis of some noval 2,4-diaryl-6-(5-methyl-1-(p-tolyl)-1H-1,2,3-triazol-4yl)pyridine derivatives. Ind J Chem 48B(06):873-876

32. Shawali AS, Abdelhamid AO (1976) Reaction of dimethylphenacylsulfonium bromide with nucleophiles. Bull Chem Soc Jpn 49:321-324

33. Ewess NF, Osman A (1980) Synthesis of heterocycles. Part II. New routes to acetylthiadiazolines and alkylazothiazoles. J Heterocycl Chem 17(8):1713-1717

34. Pokhodylo NT, Savak RD, Matiichuk VS, Obushak ND (2009) Synthesis and selected transformation of 1-[4-(4- $R$-r-methyl-1-aryl-1H-1,2,3,-triazol-1-yl) phenyl]ethanonees. Russ J Gen Chem 79:309-314

35. Fischer $\mathrm{O}$ (1876) Ueber die Einwirkung der Salpertrigen Saure auf Acetanilid. Ber Dtsch Chem Ges 9(1):463-465 